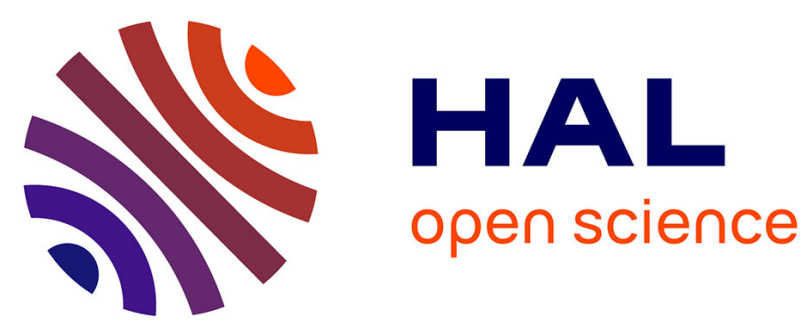

\title{
Latitudinal Beaming of Jupiter's Radio Emissions From Juno/Waves Flux Density Measurements
}

Corentin Kenelm Louis, Philippe Zarka, Keshika Dabidin-Audam, Paul-Alexandre Lampson, Fabiola P. Magalhaes, Adam Boudouma, Manilo Soares Marques, Baptiste Cecconi

\section{To cite this version:}

Corentin Kenelm Louis, Philippe Zarka, Keshika Dabidin-Audam, Paul-Alexandre Lampson, Fabiola P. Magalhaes, et al.. Latitudinal Beaming of Jupiter's Radio Emissions From Juno/Waves Flux Density Measurements. Journal of Geophysical Research Space Physics, 2021, 126 (10), pp.e2021JA029435. 10.1029/2021JA029435 . hal-03368294

\section{HAL Id: hal-03368294 https://hal.science/hal-03368294}

Submitted on 6 Oct 2021

HAL is a multi-disciplinary open access archive for the deposit and dissemination of scientific research documents, whether they are published or not. The documents may come from teaching and research institutions in France or abroad, or from public or private research centers.
L'archive ouverte pluridisciplinaire HAL, est destinée au dépôt et à la diffusion de documents scientifiques de niveau recherche, publiés ou non, émanant des établissements d'enseignement et de recherche français ou étrangers, des laboratoires publics ou privés. 


\title{
Latitudinal beaming of Jupiter's radio emissions from Juno/Waves flux density measurements
}

\author{
C. K. Louis ${ }^{1,2,3}$, P. Zarka² ${ }^{2}$ K. Dabidin' ${ }^{2}$, P.-A. Lampson² , F. P. Magalhães ${ }^{2}$, A. \\ Boudouma $^{2}$, M. S. Marques ${ }^{4}$, and B. Cecconi ${ }^{2}$
${ }^{1}$ School of Cosmic Physics, DIAS Dunsink Observatory, Dublin Institute for Advanced Studies, Dublin 15, Ireland
${ }^{2}$ LESIA, Observatoire de Paris, PSL Research University, CNRS, Sorbonne Universités, UPMC Univ. Paris 06, Univ. Paris Diderot, Sorbonne Paris Cité, Meudon, France
${ }^{3}$ Institut de Recherche en Astrophysique et Plantologie (IRAP), Universit de Toulouse, CNRS, CNES, UPS, (Toulouse), France
${ }^{4}$ Departamento de Geofsica, Universidade Federal do Rio Grande do Norte, Natal, RN, Brazil

Key Points:

- We build a processing pipeline of Juno/Waves data that includes conversion to absolute flux densities.

- We build a catalog of all Jovian radio components over the first 3 years of Juno's orbital mission.

- We derive occurrence and intensity distributions vs. observer's latitude and frequency for each component.

Corresponding author: Corentin Kenelm LOUIS, corentin.louis@dias.ie 


\begin{abstract}
The observations from the Juno spacecraft in polar orbit of Jupiter provide for the first time a complete view of Jupiter's radio emissions from all latitudes. Characterizing the latitudinal distribution of radio emissions' occurrence and intensity is a useful step for elucidating their origin. Here we analyze for that purpose the first 3 years of observations from the Waves experiment on the Juno spacecraft (mid-2016 to mid-2019). Two prerequisites for the construction of the latitudinal distribution of intensities for each Jovian radio component are (i) to work with absolute flux densities, and (ii) to be able to associate each radio measurement with a specific radio component. Accordingly, we develop a method to convert the Juno/Waves data in flux densities and then we build a catalog of all Jovian radio components over the first 3 years of Juno's orbital mission. From these, we derive occurrence and intensity distributions versus observer's latitude and frequency for each component; these will be the basis for future detailed studies and interpretations of each component's characteristics and origin.
\end{abstract}

\title{
1 Introduction
}

A complex zoo of low-frequency radio components, from $<1 \mathrm{kHz}$ to $\sim 40 \mathrm{MHz}$, is produced in Jupiter's magnetosphere. This zoo is more complex than at any other planet in the solar system. Its components have been discovered from the ground for those whose spectrum exceeds the Earth's ionospheric cutoff $\sim 10 \mathrm{MHz}$, and from space for the other ones, essentially by the Voyager $1 \& 2$ spacecraft (Carr et al., 1983; Kaiser, 1993; Zarka, $2002,2004)$. Some of the components are very intense. For example, Jovian auroral decameter radio emissions can compete with solar radio emissions (Zarka, 2007). They have been further studied phenomenologically and statistically with ground-based decameter arrays (e.g. in Nançay; Lamy et al., 2017) and with various spacecraft, in orbit around Jupiter like Galileo (Gurnett et al., 1992), flying-by like Ulysses (Stone et al., 1992) and Cassini (Zarka et al., 2004), or remotely like Wind (Aubier et al., 2000).

The emission mechanism of high-latitude components has been identified as the electron cyclotron maser instability (ECMI) and well studied (Zarka, 1998; Treumann, 2006; Louarn et al., 2017, 2018; Louis, Louarn, et al., 2020), to the point where the time-frequency morphology of these emissions can be modelled quite accurately (Hess et al., 2008; Louis, Lamy, Zarka, Cecconi, Imai, et al., 2017). By contrast, low-latitude emissions are attributed to mode conversion mechanisms producing emissions at or near the local plasma frequency, possibly on density gradients (e.g. plasma torus or magnetopause), but those are less quantitatively documented (Barbosa, 1982; Gurnett \& Scarf, 1983; Jones, 1988; Ronnmark, 1992).

All observational studies have been performed so far from low Jovian latitudes. The Earth's always remains within $\pm 4^{\circ}$ of Jupiter's equator, and the various spacecraft have remained within a few degrees of the equator, except for brief and remote observations from Ulysses (Stone et al., 1992; Kimura et al., 2008a). The Juno spacecraft, in polar orbit around Jupiter since mid-2016, provides for the first time a complete sampling of all observer's latitudes, allowing us to determine the overall emission diagram of each component as a function of the Jovian latitude (Kurth, Imai, et al., 2017). This is the main purpose of the present paper.

This determination provides new constraints (i) on the emission mechanisms (for a given radio component, the overall latitudinal beaming is the convolution of the beaming of each point source with the spatial distribution of the sources), (ii) on the distribution of the plasma density and its gradients in the Jovian magnetosphere (the availability of a reliable magnetic field model removes a large part of the uncertainties related to the topology of the magnetic field), and possibly (iii) on the location of energetic electron precipitation. Furthermore, the planetocentric distribution of radio component beaming is interesting to extrapolate to the case of exoplanets, from which one attempts to 
detect stronger analogues of Jupiter's radio emissions (see e.g. Turner et al., 2021), in order to document favourable viewing geometries.

In order to derive this overall latitudinal beaming per component, two prerequisites should be fulfilled: (1) the radio measurements from the Juno/Waves experiment, which detects radio waves around Jupiter, must be converted to absolute flux densities, and (2) these radio measurements should be sorted by Jovian radio component.

Flux density calibration has been successfully achieved for radio experiments onboard e.g. Cassini or Wind because these experiments were sensitive enough to detect the galactic radio background. Measurement of this background by a short electric dipole (of tip-to-tip length $<\lambda / 2$ ), that has a broad beam $\sim 8 \pi / 3 \mathrm{sr}$, has been well documented (Manning \& Dulk, 2001; Dulk et al., 2001) so that it provides the basis for an absolute calibration to an accuracy within a factor $\sim 2$ (Zarka et al., 2004). Spacecraft-generated interference is generally narrowband enough to be interpolated from surrounding unpolluted measurements. However, the Waves experiment on Juno (Kurth, Hospodarsky, et al., 2017) has a limited sensitivity, partly due to its shorter electric dipole compared to Cassini's or Wind's, so it cannot detect the Galactic radio background, but only Jovian signals and spacecraft-generated interference. As the latter is not sufficiently well controlled to be usable (especially in the high-frequency part of the instrument), one has to rely on the Jovian radio signals themselves to estimate flux densities from the Juno/Waves measurements. But how can we deduce measured flux densities on the basis of the signals that the instrument is supposed to map? The solution that we propose here is to match the long-term statistical averages (or peaks) of the Cassini (and Voyager) Jovian signals with those recorded by Juno in the same geometrical configuration, i.e. from low Jovian latitudes only. With the conversion table thus established, one can then turn Juno measurements performed at all latitudes into absolute flux densities. The processing and conversion are presented in Appendix A, and the processed data in flux density units are available at https://doi.org/10.25935/6jg4-mk86 (Louis, Zarka, \& Cecconi, 2021).

In Section 2, we give an overview of Jupiter's radio components. In Section 3, we briefly present the Juno/Waves experiment and its mission context. In order to study separately each Jovian radio component, we build in Section 4 a catalog of Jovian radio emission events detected in daily Juno/Waves dynamic spectra (intensity as a function of time and frequency), over the first 3 years of Juno's orbital mission. In Section 5 , based on the previous processing and catalog, we derive for the first time reference distributions of emission occurrence and intensity for each component as a function of latitude and frequency. Finally, Section 6 summarizes and discusses our results as well as prospects for subsequent studies.

Supplementary Table and Figures are provided as separate Supporting Information (SI).

\section{Jovian radio components}

Jupiter's low-frequency radio spectrum includes about half a dozen components identified in previous studies, between $\sim 1 \mathrm{kHz}$ and $40 \mathrm{MHz}$ (see review papers of Zarka, 1998, 2004, references therein, and references below). We briefly present them from low to high frequencies, as known in the pre-Juno era. Most of them show up in e.g. Figure 1 of Zarka et al. (2001), as well as on Figure 2 of the present paper displaying Juno/Waves data.

At the lowest frequencies, the so-called Quasi-Periodic (QP) bursts cover the range from $\leq 1 \mathrm{kHz}$ to $20-30 \mathrm{kHz}$. They were discovered using Voyager data (Kurth et al., 1989) and named Jovian type III bursts owing to the time-frequency drift of their lowfrequency tail. They were studied again using Ulysses radio observations and named QP bursts owing to their quasi-periodicities at $\sim 15$ and $\sim 40$ minutes (MacDowall et al., 
1993). They present strong analogies with the Terrestrial low-frequency bursts from the Earth's magnetosphere (Steinberg et al., 2004, and references therein). As them, QP bursts seem to be emitted from high-latitude (auroral), high-altitude regions and are then subject to complex propagation that generates the drifting tail (MacDowall et al., 1993; Desch, 1994; Hospodarsky et al., 2004; Steinberg et al., 2004). The superposition of the QP tails results in a continuum of emission that is partly trapped in the magnetospheric plasma cavity, and partly escaping it (Kaiser et al., 1992; Imai et al., 2017). This continuum superposes to the ubiquitous trapped nonthermal continuum radiation below $5 \mathrm{kHz}$ (Gurnett \& Scarf, 1983) and the escaping continuum radiation slightly above $5 \mathrm{kHz}$, produced by mode conversion on density gradients (that we shall not study in this paper). There is no consensus on the generation mechanism of QP bursts (CMI? mode conversion?), although it is believed that the emission is produced at or close to the local electron cyclotron frequency $\left(f_{c e}=e B / 2 \pi m_{e}\right.$, where $e$ and $m_{e}$ are the electron charge and mass and $B$ the local magnetic field amplitude). There is no consensus either on their emission mode, although O-mode is favoured (Kimura et al., 2008b, 2012). The origin of the quasi-periodicities of these bursts is thought to be related to electron bursts in the outer magnetosphere (MacDowall et al., 1993), but the precise relationship remains to be elucidated.

Overlapping with the QP range, from a few $\mathrm{kHz}$ to a few tens of $\mathrm{kHz}$, one finds a component structured in narrow bands of emission, rarely reported in the literature. It covers a frequency range higher than the escaping continuum radiation and may include the so-called narrowband electromagnetic emissions identified by Gurnett et al. (1983). It may be generated by mode conversion on plasma density gradients at the plasma frequency $\left(f_{p e}=(1 / 2 \pi)\left(N_{e}^{2} / \epsilon_{0} m_{e}\right)^{1 / 2}\right.$, where $N_{e}$ is the electronic plasma density and $\epsilon_{0}$ the vacuum permittivity) or the upper hybrid resonance frequency. Hereafter we will refer to this component as narrowband Low Frequency (nLF) emission.

At slightly higher frequencies, one finds another narrowband emission called the narrowband kilometer emission $(\mathrm{nKOM})$ in the range $\sim 60-160 \mathrm{kHz}$. It was discovered with Voyager (Kaiser \& Desch, 1980) and its source was located by Ulysses in Io's plasma torus (Reiner et al., 1993). Its emission mechanism is not known with certainty, but its source location is only compatible with emission at or near $f_{p e}$. Both the nLF and nKOM show up on dynamic spectra as fuzzy patches of emission elongated in time.

Above $\sim 20 \mathrm{kHz}$ and up to a few hundred $\mathrm{kHz}$ (typically $400 \mathrm{kHz}$, cf. Zarka et al., 2004) lies the broadband kilometric emission (bKOM), made of sporadic, often drifting features. Originating along high-latitude, auroral field lines with apex at 15-60 $R_{J}$ (1 $R_{J}=$ Jovian radius $\sim 71400 \mathrm{~km}$ ) from the planet (Ladreiter et al., 1994; Kurth et al., 1980; Imai et al., 2019; Louis, Prangé, et al., 2019), bKOM is produced on the X-mode by the CMI near the local $f_{c e}$ at distances $\geq 5 R_{J}$ away from the planet. The cause for its sporadicity is not understood.

Above $200-300 \mathrm{kHz}$ and up to a few $\mathrm{MHz}$ is the hectometric (HOM) emission. Much more steady than the bKOM emission (Zarka et al., 2004), it is observed at longitudes approximately in antiphase with bKOM (Boischot et al., 1981; Lecacheux et al., 1992). It is also definitely attributed to the CMI and has sources along auroral field lines with apex similar to or slightly lower than bKOM (Ladreiter et al., 1994; Louis, Prangé, et al., 2019).

Finally, the auroral decametric emission (DAM) covers the range from a few $\mathrm{MHz}$ to $40 \mathrm{MHz}$. It is the only low-frequency planetary radio emission detectable from the ground above the Earth's ionospheric cut-off $(\sim 10 \mathrm{MHz})$, if one omits the synchrotron emission from radiation belts at much higher frequencies $(\mathrm{GHz})$. It is produced primarily on the X-mode near the local $f_{c e}$ by the CMI (Zarka, 1998). It is connected in a complex way to the HOM, which is not simply the low-frequency extent of DAM (Imai et al., 2011). The decameter range actually includes the emission related to the main Jovian aurora, 


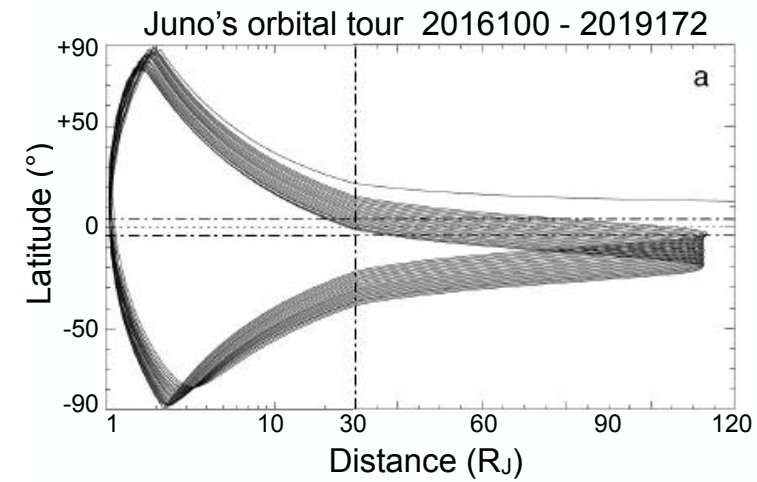

\section{Juno mission and Juno/Waves experiment}

along field lines connected to the main auroral oval (Louis, Prangé, et al., 2019) and decametric emissions induced by the interaction of the Jovian magnetic field with the Galilean satellites Io, Ganymede and Europa (Bigg, 1964; Menietti et al., 1998; Higgins et al., 2006; Marques et al., 2017; Louis, Lamy, Zarka, Cecconi, \& Hess, 2017; Zarka et al., 2018). It is possible to distinguish between these sources on the basis of the time-frequency shape of the emissions, their statistical occurrence in observer's longitude-satellite phase planes, and modelling by the ExPRES code (Louis, Hess, et al., 2019).

Juno has been performing its orbital tour since 2016/07/03. The spacecraft flys around Jupiter on polar orbits of period $\sim 53$ days, of which \pm 3 hours are spent within a distance of $5 R_{J}$ of Jupiter (and $\pm 6 \mathrm{~h}$ within $8 R_{J}$ ) around each perijove (closest approach to Jupiter). During these brief intervals, very high latitudes are sampled (up to $\pm 90^{\circ}$ ).

Figure 1 shows (a) the latitudes explored along the Juno trajectory during the first 3 years of the mission, that cover the first 20 perijoves, as a function of the distance to Jupiter, and (b) the cumulative time (in minutes) spent at each latitude.

Figure 1. (a) Jovicentric latitudes explored by Juno as a function of the distance during the interval covered by the present study, between DoY 100 of $2016(2016 / 04 / 09)$ and DoY 172 of 2019 (2019/06/21). The distance scale is logarithmic between 1 and $30 R_{J}$, and linear beyond $30 R_{J}$. The processing pipeline described in Appendix $\mathrm{A}$ is based on data recorded at latitudes within $\pm 4^{\circ}$ of the equator (magnetic latitude within $\pm 14^{\circ}$ ) and beyond $30 R_{J}$ (these limits are indicated by dash-dotted lines). (b) Time (in minutes) spent by Juno within each $1^{\circ}$ bin of latitude during the same 3-year interval (solid line). The dashed line is the time spent by Juno at distances $\geq 30 \mathrm{R}_{J}$. The thick line, within $\pm 4^{\circ}$ of latitude, corresponds to the data used for our processing pipeline (the peak around $+10^{\circ}$ corresponds to Juno's approach trajectory).

The orbital period is not a multiple of the planet's rotation period, so that different longitudes are sampled, orbit after orbit. Juno/Waves thus observes Jovian radio components from all points of view. Juno spins around the axis of its high-gain antenna, making one revolution per $30 \mathrm{~s}$ (with fluctuations of a few percent along the tour).

The Waves instrument onboard Juno consists of 2 receivers connected to an electric dipole antenna (made of two $2.8 \mathrm{~m}$ monopoles) and a magnetic search coil (Kurth, Hospodarsky, et al., 2017). We are interested here in electric measurements only (magnetic measurements are restricted to frequencies $\leq 20 \mathrm{kHz}$ ). Together, the two receivers cover the full spectral range of all Jovian radio components and plasma waves. Each receiver samples two frequency bands. The Low Frequency Receiver (LFR) samples the 
bands LFR-low from $\sim 50 \mathrm{~Hz}$ to $20 \mathrm{kHz}$, and LFR-high from $\sim 20$ to $140 \mathrm{kHz}$. The High Frequency Receiver (HFR) samples the bands HFR-low from $\sim 140 \mathrm{kHz}$ to $3 \mathrm{MHz}$, and HFR-high from $\sim 3$ to $41 \mathrm{MHz}$. The receivers record waveforms of the sampled electric signal below $3 \mathrm{MHz}$, from which low-resolution (so-called survey mode) and highresolution (so-called burst mode) dynamic spectra are produced. Above $3 \mathrm{MHz}$, the survey mode data are recorded in swept frequency analyzer mode. Survey-mode data are continuously recorded along Juno's orbit, a full spectrum being produced every $1 \mathrm{~s}$ to $30 \mathrm{~s}$ (the resolution of $1 \mathrm{~s}$ is always used around perijoves, and actually over a large fraction of the time on the rest of the orbits). The spectral resolution depends on the receiver and on the observation mode. In survey mode, a spectrum $50 \mathrm{~Hz}-41 \mathrm{MHz}$ is divided in 126 frequency channels, 110 of which are above $1 \mathrm{kHz}$ (which is the minimum frequency addressed in this paper).

An examination of the Juno/Waves survey data (see Appendix A) shows that the HFR-high band is polluted by narrowband interference, spacecraft- or instrument-generated. The HFR-Low sub-band has a low sensitivity and is able to detect Jupiter's signals only within a few (but crucial) hours around the perijoves, returning only noise and interference at greater distances from Jupiter (see Figure 2). Level jumps ( $\mathrm{dB}$ to tens of $\mathrm{dB}$ ) are observed between sub-receivers. For these reasons, we processed the Juno/Waves data as described in Appendix A to correct these problems at best before converting the measurements into absolute flux densities. In this paper we used survey-mode "PDS v1" data. But our processing can similarly be applied to the recalibrated "PDS v2" data (or any subsequent recalibrated data set) and will lead to the same flux densities, only with a different conversion table.

\section{Catalog of Jovian radio components}

Little directional information is provided by Juno/Waves (cf. Imai et al., 2017). Thus, in order to distinguish between the Jovian radio component in the frame of a large scale statistical study, it is necessary to identify and separate them in dynamic spectra. Automated recognition based on machine-learning techniques will likely be efficient soon, but in the meantime (and also to train these future recognition algorithms), we created "manually" a catalog of Jupiter's radio components from daily (24 hours) dynamic spectra of processed flux densities. The method is similar to that defined in Marques et al. (2017) (see in particular Appendix A of that paper). The radio components were visually identified according to their time-frequency morphology, and then manually encircled by contours and labelled, using a dedicated program that records the coordinates of the contours and the label of each emission patch. Figure 2 displays two typical $24 \mathrm{~h}$ processed dynamic spectra with their catalogued emissions.

In total, $\sim 5800$ contours were drawn. Catalogued components include: nKOM $(\sim 2000$ events $), \mathrm{nLF}(\sim 1200$ events), QP $(\sim 1150$ events $)$ and satellite-induced DAM emissions $(\sim 1350$ events, of which 1085 are Io-induced DAM events). While nKOM and nLF patches can be identified individually (cf. Figure 2), QP bursts often form a quasicontinuum of emission at the lowest frequencies. Thus, we have chosen to include in a single daily contour the region of the dynamic spectrum containing QP bursts and nonthermal continuum (with a variable maximum frequency separating QP from nLF or bKOM), that will allow us to analyze in detail the content of these regions (e.g. burst periodicities) in further works. Satellite-induced emissions were identified by comparing the Juno/Waves dynamic spectra with simulations performed using the ExPRES code (Louis, Hess, et al., 2019). The simulations allow to predict the occurrence and shape of time-frequency DAM arcs induced by the interaction of Io, Ganymede and Europa with Jupiter's magnetic field (as in Louis, Lamy, Zarka, Cecconi, \& Hess, 2017; Louis, Lamy, Zarka, Cecconi, Imai, et al., 2017), that can then be identified in the data. The latest Jovian magnetic field model JRM09 (Connerney et al., 2018) was used. All the simulation data are available at https://doi.org/10.25935/KPGE-ZB59(Louis, Cecconi, \& Loh, 2020). In 

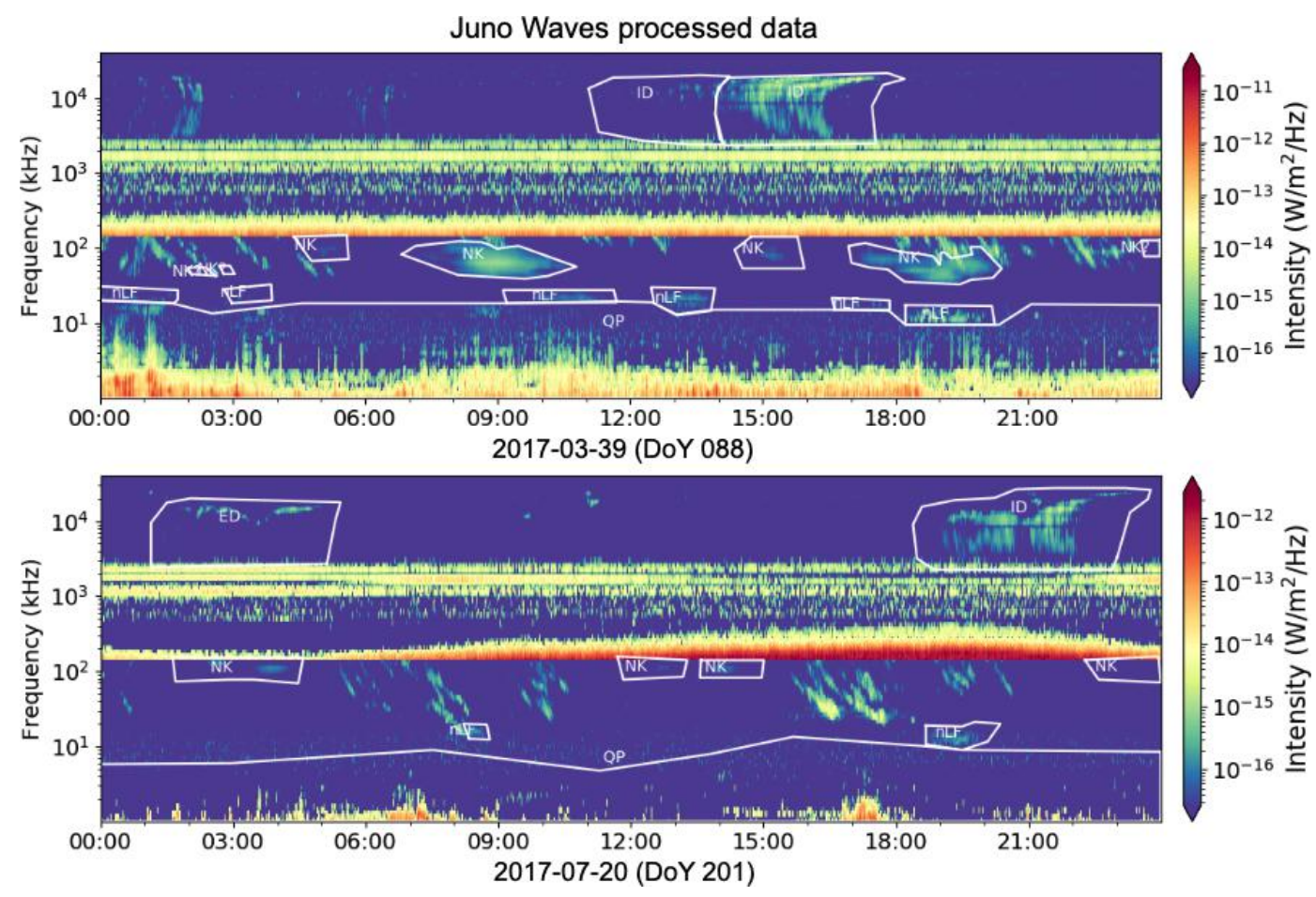

Figure 2. Example of two 24-hour dynamic spectra of processed Juno/Waves data (2017/03/29 and 2017/07/20, i.e. resp. DoY 88 and 201 of 2017). Catalogued events are indicated by the white contours and labels. The ubiquitous drifting features below $150 \mathrm{kHz}$ are bKOM, emission patches and arcs not otherwise identified above $3 \mathrm{MHz}$ are auroral $\mathrm{HOM}$ and DAM emissions.

the catalog these emissions are identified by a two-letter code: the first letter is I, G or $\mathrm{E}$ (for Io, Ganymede or Europa) and the second one is A, B, C, or D (following the usual identification of the corresponding Jovian radio source in the northern - A,B - or southern - C,D - hemisphere, and on the western - B,D - or eastern - A,C - limb). Far from the perijoves, satellite-induced radio emissions show up with a good contrast over auroral DAM, and are thus recognizable with confidence. Close to perijoves $(\sim \pm 3 \mathrm{~h})$, due to the proximity of the planet and the high speed of the spacecraft $(>50 \mathrm{~km} / \mathrm{s})$, satelliteinduced emissions have an unusual time-frequency shape and are drowned in auroral DAM, thus their unambiguous identification becomes impossible.

As the background is very close to zero, the contours do not need to follow closely the shape of the emissions. They must only separate the components from each other. Then, the contents of selected contours can be included or excluded to build time series, histograms of intensities or any other derived quantity, including or excluding any labelled component. The contours are used as masks to select or exclude any component. This explains how we can select and study bKOM, HOM or auroral- (i.e. satellite-independent) DAM. These components have not been explicitly catalogued because they are the most frequent emissions in their respective frequency range. They can be selected and studied by excluding all other components and restricting to the appropriate frequency range. For example, excluding $\mathrm{nKOM}, \mathrm{nLF}$ and $\mathrm{QP}$ in the range $1 \mathrm{kHz}-1 \mathrm{MHz}$ (in practice $1 \mathrm{kHz}-140 \mathrm{kHz}$ as Waves has a lower sensitivity in the range $140 \mathrm{kHz}-3 \mathrm{MHz}$ ) allows to select the bKOM component only. 
Note that in the few hours around perijoves, Juno passed through regions where it crossed auroral radio sources (bKOM, HOM and DAM - cf. Kurth, Imai, et al., 2017; Louis, Prangé, et al., 2019). All the emissions catalogued as radio source crossings are labeled specifically ('SRC') in the catalog, so that they can be studied separately. We excluded them from our statistical study below in order to avoid normalization issues.

\section{Latitude-frequency distributions of occurrence and intensity per Jo- vian radio component}

With the processed Juno/Waves data converted to flux density units (see Appendix A), combined with the ability to study each radio component separately using our catalog (see Section 4), we built here for the first time the observed occurrence probabilities and flux density distributions as a function of observer's latitude and frequency for each Jovian radio component. From the contours recorded in the catalog, it is straightforward to extract the frequency ranges covered by each component (using the masks in the catalog to select wanted components or exclude unwanted ones). They are listed in Table 1. Within these frequency ranges, unselected data are set to zero, then we build an histogram of the intensities of selected data in each frequency channel. This histogram has the shape of a Gaussian distribution plus a higher intensity tail. The Gaussian distribution contains the residual background in the selected data, whereas the tail characterizes the intensities of the component studied. Examples of such histograms are displayed in Figure S2 (Supporting Information). The lower limit of the tail can be easily identified in each case from the departure from the fitted Gaussian distribution. This lower limit is taken as the threshold under which all selected intensity values are set to zero. The remaining nonzero data truly characterize the intensity distribution of the studied component.

The auroral HOM and DAM only differ by the spectral ranges covered, and the limit and connections between these two components are not clear yet (Imai et al., 2011). We will therefore study them together. The frequency ranges of the Ganymede-DAM and Europa-DAM emissions are similar to those of the Io-DAM, but we will not study here their latitudinal distributions because too few events have been detected yet. Finally, we will not attempt to separate the nonthermal continuum from QP bursts tails, but background subtraction (see Appendix A) likely reduces the contribution of the nonthermal continuum to processed data. We then end up with the 6 components listed in Table 1. For each component and each frequency in the corresponding range, the thresholds determined from the histograms of intensities (like in Figure S2, Supporting Information) are tabulated in Table S1 (Supporting Information).

Table 1. Frequency ranges covered by each component. The limits in parentheses fall into the HFR-low band and thus only concern the few events detected near the perijoves. For our statistical study, we exclude the HFR-low band thus the frequency ranges used are those not in parentheses.

\begin{tabular}{c|c}
\hline Radio component & Minimum - Maximum frequency \\
\hline QP & $1-140(900) \mathrm{kHz}$ \\
nLF & $1.3-130 \mathrm{kHz}$ \\
nKOM & $12-140(250) \mathrm{kHz}$ \\
bKOM & $1-140(800) \mathrm{kHz}$ \\
HOM \& DAM (auroral) & $(0.14) 3.5-41 \mathrm{MHz}$ \\
Io-DAM & $(0.14) 3.5-40 \mathrm{MHz}$ \\
\hline
\end{tabular}


To derive the occurrence probability versus the latitude and the frequency, for each Jovian radio component, we counted the number of measurements above the threshold per Juno/Waves channel and per $1^{\circ}$ latitude bin, and we divided it by the total number of measurements recorded in that latitude bin. The resulting occurrence distributions are displayed in Figure 3 as a function of the magnetic latitude of the spacecraft and of the frequency. Similar plots for the centrifugal and Jovicentric latitudes of the spacecraft are displayed in Figures S3 and S4 (Supporting Information). Figure 4 displays the intensity distribution of each component as a function of frequency and magnetic latitude. In each bin, the mean of all measurements that are above the threshold is computed. Bins with no measurement above the threshold are set to an arbitrary low value. On these two figures, gaps at fixed frequencies are due to interference. Vertical structures at low latitudes result from sporadic events occurring while the spacecraft latitude changes slowly (see below). Occurrence and intensity distributions are very complementary: for example, a radio component may be sporadic, with a low occurrence probability, but intense whenever present, and thus show up with a low occurrence probability and a high intensity. All other combinations are possible.

We compare below the obtained distributions in the three latitude systems (magnetic, centrifugal and Jovicentric), attempting to determine which one organizes better the data for each radio component. Based on Connerney et al. (2018), Jupiter's magnetic equator is tilted by an angle $\alpha=10.31^{\circ}$ relative to the Jovicentric equator, while the centrifugal equator is inclined relative to the Jovicentric equator by an angle $\beta=$ $\frac{2}{3} \alpha$ (Hill et al., 1974). As Figures 3 and 4 stack data over 3 years and 20 orbits, a better data organization should correspond to sharper features with a higher contrast in the panels of Figures 3 and 4. However, as Juno's Jovicentric latitude varies slowly along its orbits (cf. Figure 1) sporadic bursts will appear as intense vertical lines in the distributions in Jovicentric latitude (Figure S4). Since the centrifugal latitude oscillates by $\pm 6.9^{\circ}$ with Jupiter's rotation, and the magnetic latitude by $\pm 10.3^{\circ}$, these oscillations tend to blur the emission patches from Jovicentric to centrifugal to magnetic latitude systems. Thus if we observe a higher contrast in magnetic latitude, it indicates that the corresponding component is physically better organized in this system (this is expected for auroral emissions produced at $f_{c e}$ along magnetic field lines). If the contrast is higher in centrifugal latitude, it suggests that either this latitude system or the magnetic one better organizes the corresponding component (low latitude emissions produced at $f_{p e}$ are expected to depend more on centrifugal latitude). Finally, if the contrast is higher in Jovicentric latitude, no firm conclusion can be drawn as it might be an orbital/temporal effect as described above.

Below, we describe qualitatively the 6 panels of Figures 3 and 4 (with sometimes comparison to Figures S3 and S4, Supporting Information), that are further discussed in the next section, leaving detailed analyses and modeling of the observed features to future works.

\section{1 nKOM component}

nKOM occurrence and mean intensity are displayed on panel a of Figures 3 and 4. Emission above $60 \mathrm{kHz}$ is beamed within the latitude range $-50^{\circ}$ to $+35^{\circ}$ of the equator and is asymmetric between North and South. Minimum occurrence occurs around $-10^{\circ}$, and maximum occurrence at mid-latitudes $\left(-50^{\circ}\right.$ to $-35^{\circ}$ in the South, and $+15^{\circ}$ to $+35^{\circ}$ in the North). In addition, patches of higher latitude emission show up in the range $30-60 \mathrm{kHz}\left(-80^{\circ}\right.$ to $50^{\circ}$ in the South, and $+30^{\circ}$ to $+80^{\circ}$ in the North). The midlatitude patches seem better organized in magnetic latitude, whereas the higher latitude $30-40 \mathrm{kHz}$ patches seem better organized in centrifugal latitude (see Figure S3, Supporting Information). Figure 4a also shows that even if the emission is less frequent at low latitude, it can be as or more intense than at high latitude. 

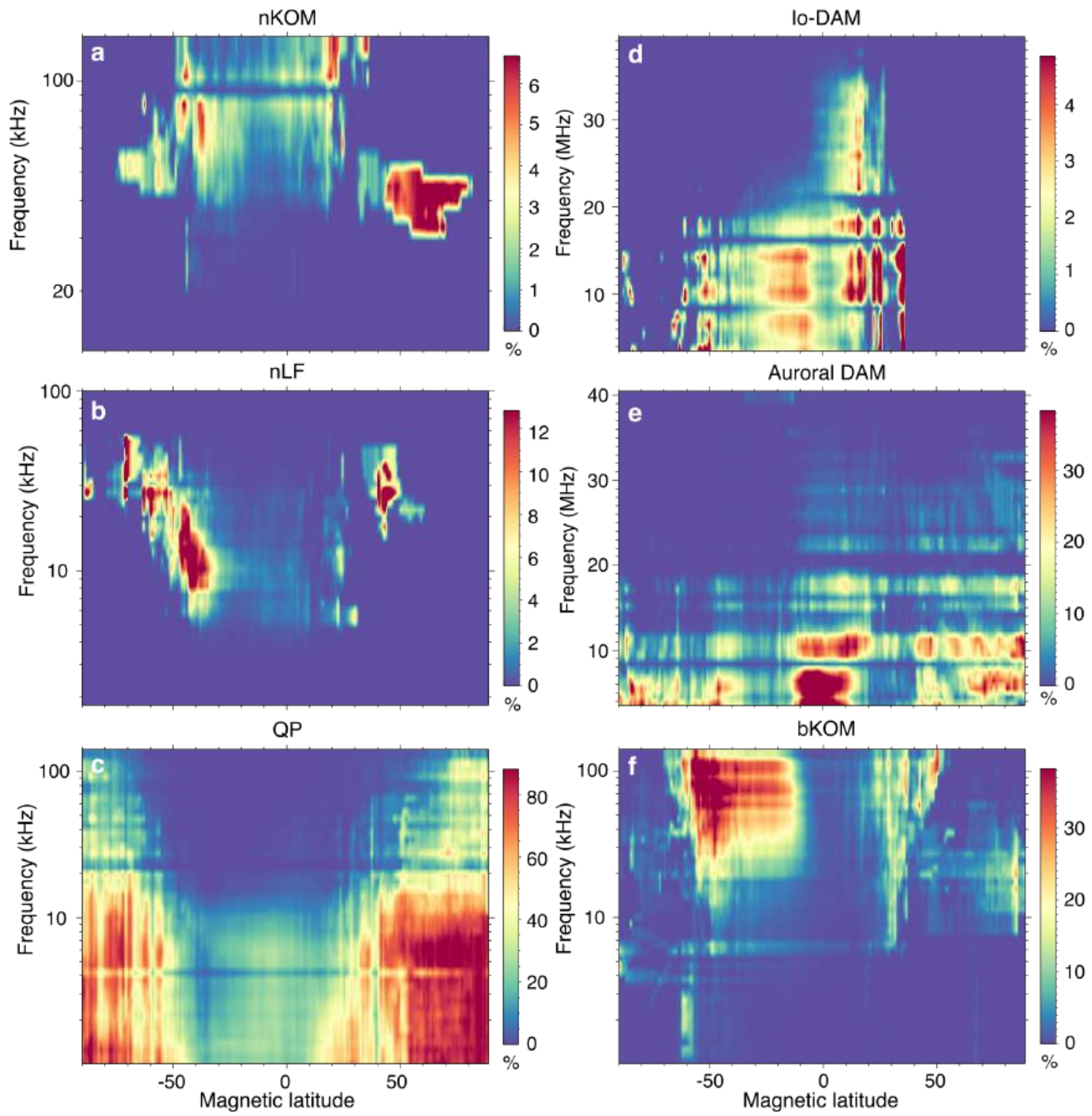

Figure 3. Occurrence probability of each Jovian radio component as a function of Juno's magnetic latitude and frequency. The latitude bins are $1^{\circ}$ wide and the frequencies are those of Juno/Waves channels. (a) nKOM, (b) nLF, (c) QP, (d) Io-DAM, (e) auroral DAM, (f) bKOM (see Table 1).

\section{2 nLF component}

nLF occurrence and mean intensity are displayed on panel b of Figures 3 and 4. The distribution is reminiscent of nKOM at first look, with a near-equatorial minimum, mid-latitude maximum occurrence and high-latitude patches, but there are important differences: $\mathrm{nLF}$ covers lower frequencies (mostly $<60 \mathrm{kHz}$ ). Its broad minimum $\left(-25^{\circ}\right.$ to $+15^{\circ}$ ) reaches much lower occurrences than nKOM at low latitudes. The North-South asymmetry is more pronounced than for nKOM, with much more southern emission, down to $-85^{\circ}$ to $-90^{\circ}$. The mid-latitude emission is in the range $5-50 \mathrm{kHz}$, whereas high-latitude patches $\left(-85^{\circ}\right.$ to $-50^{\circ}$ and $+30^{\circ}$ to $\left.+60^{\circ}\right)$ are in the range $20-50 \mathrm{kHz}$. Mid-latitude emission seems better organized in centrifugal latitude (see Figure S3, Supporting Informa- 

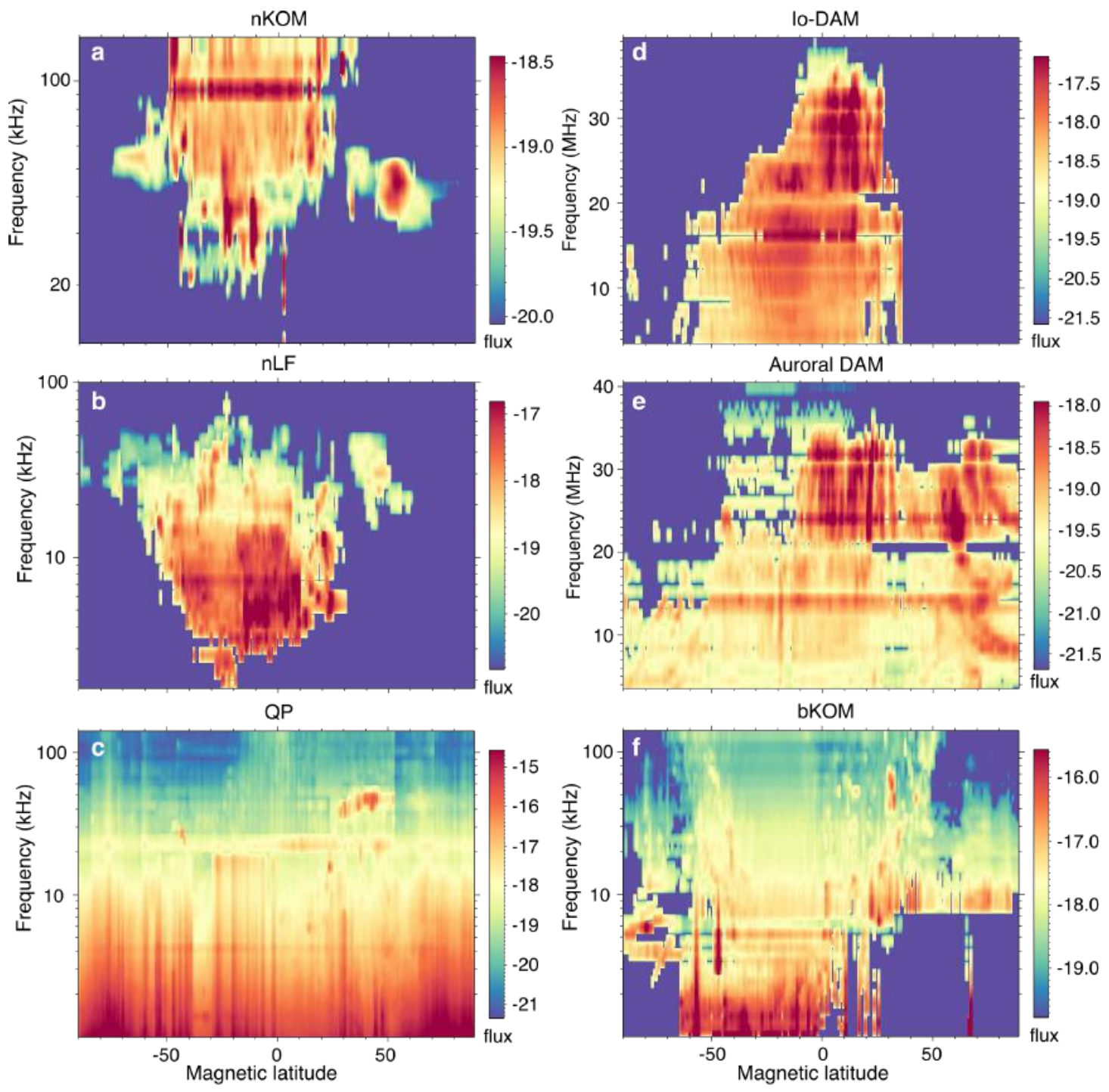

Figure 4. Intensity normalized to a distance of $1 \mathrm{AU}$ (in $\mathrm{W} / \mathrm{m}^{2} / \mathrm{Hz}^{2}$ ) of each Jovian radio component as a function of Juno's magnetic latitude and frequency. The latitude bins are $1^{\circ}$ wide and the frequencies are those of Juno/Waves channels. (a) nKOM, (b) nLF, (c) QP, (d) Io-DAM, (e) auroral DAM, (f) bKOM (see Table 1).

tion), whereas the higher latitude emission around $30-40 \mathrm{kHz}$ seems better organized in magnetic latitude: this is opposite to the nKOM situation.

Regarding the intensity of the nLF emission, Figure $4 \mathrm{~b}$ shows that the emission at low latitude is more intense than nKOM, and more intense than high-latitude nLF, even if less frequent. We note a clear trend of increasing intensity with decreasing frequencies.

\subsection{QP component}

QP occurrence and mean intensity are displayed in Figures 3c and 4c. It has a low occurrence and frequency extent $(\leq 10-20 \mathrm{kHz})$ at low latitudes, as was observed by Cassini, Voyager and Galileo. Conversely, the occurrence probability increases considerably (up 
to $>80 \%=$ highest of all Jovian radio components) toward high latitudes, up to $\pm 90^{\circ}$, and its frequency extent increases as well up to $\geq 150 \mathrm{kHz}$. It shows an asymmetric distribution, being more intense and extended over a broader latitude range in the northern hemisphere. The minimum occurrence, that never quite reaches zero, covers the range $-45^{\circ}$ to $+15^{\circ}$, i.e. it is centered around $-15^{\circ}$. QP looks more confined to high latitudes in the magnetic latitude system, that seems consequently to better organize the data. Figure 4c shows that QP is almost uniformly intense at all latitudes, and clearly more intense below $20 \mathrm{kHz}$ than above. Below $5 \mathrm{kHz}$ it may include some trapped continuum emission, and escaping continuum below $\sim 10 \mathrm{kHz}$. Note that an emission catalogued as QP (patch at $\sim 50 \mathrm{kHz}$ and $+30^{\circ}$ to $+50^{\circ}$ latitude, not visible in the occurrence Panel Figure $3 \mathrm{c}$ ) seems to be actually $\mathrm{nLF}$.

\section{4 bKOM component}

bKOM occurrence and mean intensity are displayed in Figures $3 \mathrm{f}$ and $4 \mathrm{f}$. They display an extremely asymmetric pattern, with peaks at about $-45^{\circ}$ and $+30^{\circ}$ in the range $5-140 \mathrm{kHz}$, covering a broader latitude range with increasing frequency. bKOM has the second highest occurrence of all Jovian radio components $(>60 \%)$. In addition to the main component, an extent toward very high latitudes shows up in the range 10-60 kHz. The deep equatorial minimum is centered around $+10^{\circ}$ with asymmetrical gradients on its edge (softer to the South, steeper to the North). bKOM occurrence looks sharper in centrifugal latitude (see Figure S3, Supporting Information). Figure $4 \mathrm{f}$ shows that above $\sim 10 \mathrm{kHz}$, the emission is more intense at mid-latitudes than at low-latitudes. The emission is more intense at low frequencies where its occurrence is lower, i.e. it is both more sporadic and more intense at low frequencies. Below a few $\mathrm{kHz}$, it is likely contaminated by QP emissions.

\subsection{Auroral HOM and DAM components}

HOM \& DAM occurrence and mean intensity are displayed in Figures 3e and 4e. Only observations at a distance $\leq 45 R_{J}$ were included in these plots because at larger distances, auroral DAM fades out and only Io-DAM emissions remain visible in the HFRHigh band (see Louis, Lamy, Zarka, Cecconi, Imai, et al., 2017). Horizontal gaps are due to interference. The apparent organization of the data slightly favours magnetic latitude. HOM emission, up to $\sim 6-8 \mathrm{MHz}$, appears as the Jovian radio component most symmetrically distributed in latitude. Its intensity is nearly uniform at all latitudes, and it may be slightly underestimated because a fraction of HOM signal may have been included in the subtracted background. Its occurrence remains high up to $90^{\circ}$, with two relative minima in the ranges $-30^{\circ}$ to $-20^{\circ}$ and $+20^{\circ}$ to $+30^{\circ}$. HOM occurrence is significantly higher than DAM's. DAM consists of a population or time-frequency arcs not exceeding $20 \mathrm{MHz}$ detected over a broad range of latitudes, plus arcs reaching $\sim 40 \mathrm{MHz}$ detected in a more restricted latitude range (low latitudes and northern hemisphere only). Figure 4e shows that the high-frequency emissions $(>20 \mathrm{MHz})$ are one order of magnitude more intense than the low-frequency ones. No intense northern (high-frequency) emission seems to be detected below $\sim-10^{\circ}$. The population of low-frequency $\operatorname{arcs}(<$ $20 \mathrm{MHz}$ ) is reminiscent of the so-called "lesser arcs" identified in Voyager radio measurements (Boischot et al., 1981; Boischot \& Aubier, 1981; Alexander et al., 1981), which have not yet received a clear physical interpretation. The North-South asymmetry of high-frequency arcs is likely related to the higher maximum amplitude of the planetary magnetic field in the northern hemisphere, with more electrons mirrored in the North and more precipitating in the southern hemisphere. The highest frequencies are observed from low latitudes, which may be due to a beaming effect (Galopeau \& Boudjada, 2016; Louis, Lamy, Zarka, Cecconi, Imai, et al., 2017) that deserves further investigation. 


\subsection{Io-DAM component}

Io-DAM occurrence and mean intensity are displayed on Figures 3d and 4d. As noted above, identification of satellite-induced emissions within auroral DAM based on ExPRES simulations is almost impossible too close to perijoves, and this is the only time when the highest latitudes are explored. This explains the apparently zero occurrences at high northern and southern latitudes, but a small fraction of the emissions identified as auroral DAM are certainly satellite-induced DAM emissions. Both centrifugal and magnetic latitude systems do as well for organizing the emissions, but the southern high-latitude part looks sharper in magnetic latitude. Occurrence is very asymmetrical, from about $-70^{\circ}$ to $+40^{\circ}$, and it is higher at its mid-latitude edges (clearly different from the auroral DAM). Higher frequencies are observed in the North (in the range $0^{\circ}$ to $30^{\circ}$ ). These high frequencies are not observed south of $-5^{\circ}$. The occurrence pattern shows relative minima $0^{\circ}$ and $-35^{\circ}$, i.e. symmetrical around $-15^{\circ}$. Even more clear than for the Auroral DAM, Figure 4d shows a steep gradient of intensity toward high-frequencies with emissions $>20 \mathrm{MHz}$ being one order of magnitude more intense than emissions $\leq 10 \mathrm{MHz}$.

Note that very few data are available for Europa- and Ganymede-induced radio emissions, that show essentially the same organization as Io-DAM.

\section{Summary, discussion and prospects}

We have developed a pipeline for processing the Juno/Waves data and converting them into absolute flux densities (see Appendix A), based on Jupiter radio emissions observed from low latitudes. The method consists in comparing emission statistics (50\% occurrence and 1\% occurrence spectra) derived from Cassini observations at Jupiter (Zarka et al., 2004), complemented by Voyager measurements from (Zarka, 1992) (Figure S1, Supporting Information), with similar statistics derived from Juno/Waves measurements taken in similar observation configurations ( $\mid$ Jovicentric latitude $\mid \leq 4^{\circ} \&$ distance $\geq 30 R_{J}$ ). We found that it was necessary to pre-process Juno data in order to isolate Jupiter signals from spacecraft- and/or instrument-generated interference. Pre-processing includes an FFT filtering of "PDS v1" data (as provided by the NASA/PDS, and then resampled on a regular time grid of e.g. 1 s to fulfilled the Nyquist-Shannon sampling criterion), frequency channel per frequency channel, eliminating all signals not modulated by the spacecraft rotation (outside of $(N \pm 0.15) / 30 \mathrm{~Hz}$ windows, with $N=1$ to 8 ), followed by subtraction of a reference background computed over the first three years of the mission. The processed data allowed us to build a frequency-dependent conversion table, which can then be used to convert any Juno/Waves dataset into absolute flux densities, after FFT filtering and background subtraction. A specific procedure has been developed for the HFR-low band because of its very low sensitivity, and a constant conversion curve is used below $5 \mathrm{kHz}$ to bypass the presence of the trapped nonthermal continuum (see Appendix A).

Flux densities can be further normalized to a fixed observer's distance if needed. This is useful only for statistical studies (such as the one we performed about the latitudinal distribution of the detected emissions), but not for local studies (e.g. of the wave electric field in the sources).

The estimated error is within a factor 2 in the LFR-low band above $5 \mathrm{kHz}$, and in the LFR-high and HFR-high bands, and likely twice as large in the HFR-low band and below $5 \mathrm{kHz}$.

Then, we have built a catalog of all Jovian radio components over the first three years of Juno's orbital mission by identifying visually and drawing contours in the timefrequency plane around the emissions of less frequent type in each spectral band. This allows us to study independently each component by selecting or excluding emission within contours. We have determined an intensity threshold per frequency (Figure S2 and Ta- 
ble S1, Supporting Information) below which measurements are set to zero, which allowed us to characterize the occurrence of Jovian emission in each Juno/Waves measurement ( 1 if above the threshold, 0 if below it). Combining the processing, the catalog and the threshold information, we could build maps of occurrence probability and mean intensity (of measurements above the threshold) for each Jovian radio component, as a function of latitude and frequency (Figures 3 and 4, and S3 \& S4). Thanks to its polar orbits, Juno provides for the first time a view of Jupiter's radio emissions from all observer's latitudes.

In the present paper we limited ourselves to a first qualitative analysis of these latitudefrequency maps, the main conclusions of which are:

1. all low-frequency components (nLF, nKOM, bKOM, QP) display an occurrence minimum near the equator, and maxima at mid-latitudes, except the QP bursts occurrence that maximizes at $\pm 90^{\circ}$;

2. all radio components except HOM have a highly asymmetric occurrence in latitude; nLF, nKOM, bKOM and QP have their overall latitude-frequency pattern shifted to the South; the minimum occurrence of bKOM is centered around $+10^{\circ}$ latitude (consistent with Kimura et al., 2008b), with asymmetric gradients on both sides;

3. nLF and bKOM show higher occurrences in the southern hemisphere;

4. conversely, QP bursts have a higher occurrence in the northern hemisphere, with a larger extent towards lower latitudes in the North; their occurrence is low at low latitudes, and increases toward higher frequencies at high latitudes ; but QP bursts are almost uniformly intense at all latitudes;

5. bKOM occurrence has high-latitude extents at $10-60 \mathrm{kHz}$;

6. nLF and nKOM occurrences have high latitude extents (up to $\pm 80^{\circ}$ ) at resp. 20$50 \mathrm{kHz}$ and 30-60 kHz; these extents have similar morphologies, very different from that of bKOM; they might belong to a unique component;

7. the occurrence of nLF and nKOM fall to zero at the highest northern and southern latitudes (this is also the case for Io-DAM but we rather attribute it to a selection effect);

8. auroral DAM and Io-DAM reach higher frequencies in the North than in the South; high-frequency emission is absent south of $-5^{\circ}$ to $-10^{\circ}$ suggesting that the northern emission is not detected from the southern hemisphere (and vice-versa?);

9. by order of decreasing occurrence, we find the QP bursts, bKOM, HOM, auroral DAM and $\mathrm{nLF}$, and finally nKOM and Io-DAM.

10. Aurora HOM and DAM, Io-DAM, and QP components are better organized in magnetic latitude. nLF and bKOM are better organized in centrifugal latitude. For nKOM it is difficult to favour one of these two systems upon the other.

Not all these observations are easy to interpret, but we can make a few remarks and propose some directions.

Point (1) could bear a different meaning for the various low-frequency Jovian radio components. For nKOM and possibly nLF (together with point 10), it is consistent with the emission being produced near the local plasma frequency in Io's torus, or at the plasmasphere or plasma sheet boundaries (see e.g. Divine \& Garrett, 1983); the peak mid-latitude occurrences of nKOM and nLF (points 1,7) could be consistent with emission beamed along density gradients; this will be the subject of a subsequent study. We note that $\mathrm{nLF}$ is frequent in Juno/waves data while it was rarely observed from the equatorial regions (Gurnett et al., 1983). This is consistent with the distribution of Figures 3b. But note however that we did not observe series of bands as regularly organized as in Gurnett et al. (1983). 
For bKOM, that is undoubtedly auroral (Ladreiter et al., 1994; Imai et al., 2019; Louis, Prangé, et al., 2019), Point (1) could be interpreted as the result of refraction on the equatorial plasma (as suggested by Kurth et al., 1980), which deserves ray-tracing studies (Kimura et al., 2008b). This is also supported by the fact (point 10) that bKOM seems better organized in centrifugal latitude than in magnetic latitude (although bKOM source locations are organized along magnetic field lines). For QP bursts (points 1,4), the beaming towards high latitudes has been attributed to propagation effects from high latitude sources (MacDowall et al., 1993; Desch, 1994; Hospodarsky et al., 2004). We find here $(9)$ that the occurrence of QP reaches extremely high values $(>80 \%)$ at high latitudes, and that it seems better organized in magnetic latitude, revealing a very active auroral-related process.

The observed North/South asymmetry (points 2,3) is likely related in fine to the asymmetric Jovian magnetic field, via the organization of the magnetospheric plasma that it governs, but no simple explanation is available. The asymmetry of the auroral and Io-DAM frequency ranges (8) is easier to interpret because the emission is produced by the CMI at/near the local $f_{c e}$ (which also explains point 10), due to the fact that the Jovian magnetic field reaches higher amplitudes in the northern hemisphere (Connerney et al., 2018). The larger occurrence in the northern hemisphere is less easy to explain. The fact that emission produced in one hemisphere is not observable from the other one beyond a few degrees of opposed latitude, in spite of ExPRES predictions, was noted by Louis, Lamy, Zarka, Cecconi, Imai, et al. (2017). It was possibly attributed to the model of Galopeau and Boudjada (2016) of an oblate emission cone produced by the CMI when taking into account the different orientations of the magnetic field and its gradient in the sources, and leading to a cone opening much smaller in the plane containing $\mathbf{B}$ and $\nabla \mathbf{B}$ (the meridian plane in the case of a dipolar field) than in the perpendicular direction.

The occurrence of HOM emissions (below 6-8 MHz) significantly higher than that of auroral-DAM (Point 9), especially in the southern hemisphere, associated with the fact that HOM emissions seem less intense, question the relationship between these two components that one would tend to consider as the low- and high-frequency parts of the same emission (Imai et al., 2011).

Finally the occurrence of Io-DAM emissions (Point 9), the lowest of all components, must be compared to that of potentially visible emissions. Comparing Io's orbital period of $42.5 \mathrm{~h}$ to the typical duration of Io-induced arcs (tens of minutes to a few hours, Marques et al., 2017), we expect Io-DAM emission to be detected 1-5\% of the time if it is permanent, less if it is not. The observed occurrences thus support permanent emission, as inferred independently by Louis, Lamy, Zarka, Cecconi, Imai, et al. (2017).

More detailed studies of each component are out of the scope of the present paper. They will be the subject of further works. In particular, studies based on the catalog and absolute flux densities should include:

- occurrence and intensity variations as a function of the observer's longitude (socalled Central Meridian Longitude) and the frequency, in the line of Imai et al. (2011). The latter authors studied such variations at low latitudes from Cassini data, and put constraints on the relative internal (rotational) and external (solar wind) control of the auroral emissions. The same study can be performed from Juno data combining in addition the information about the latitude, and extending the spectral range up to $40 \mathrm{MHz}$ (whereas Cassini radio measurements are limited to $\leq 16.1 \mathrm{MHz}$ (Gurnett et al., 2004)).

- occurrence and intensity variations as a function of the observer's Local Time, in order to determine if an asymmetry exists between the dawn and the dusk sides of the planet, and comparison to the distribution of UV auroras. 
Of course, each Jovian radio component should be studied separately, and their statistical properties compared. The dataset studied in the present paper is limited to $\sim 3$ years, but it will reach $\sim 9$ years at the end of the Juno extended mission, increasing the statistical basis by a factor $>3$. This should allow us to investigate long-term solar wind effects, variations of the solar EUV flux, and seasonal variations such as done for the Saturnian Kilometric Radiation by, e.g., Nakamura et al. (2019). We expect that this further characterization of the Jovian radio components will help understanding their origin, beaming and relations between components.

\section{Appendix A Flux density estimation of Juno/Waves}

As explained in section 1, we present in this Appendix a method to convert Juno/Waves measurements ("PDS v1" data set, but the method also applies to recalibrated "PDS v2" data) into absolute flux densities. This processing of the data provided by the PI team is primarily done by comparison with Cassini observations of Jupiter taken during the flyby of 2000-2001, which were themselves calibrated against the galactic radio background in the range $3.5 \mathrm{kHz}-16.1 \mathrm{MHz}$, with an additional validation via comparison with ground-based observations (Zarka et al., 2004). In order to characterize the spectrum of Jupiter's low-frequency radio components with Cassini, 21 intervals of duration 1 to 9 Jovian rotation periods each (the Jovian rotation period is $9 \mathrm{~h} 55.5 \mathrm{~min}$ ) were selected during the 6 months surrounding Jupiter's closest approach by Cassini, one or several radio components being active during each interval. Average and peak spectra were computed over these intervals. Cassini remained within $\pm 4^{\circ}$ of Jovicentric latitude, i.e. within $\pm 14^{\circ}$ of Jovimagnetic latitude, and farther than $137 R_{J}$ from Jupiter.

In order to use Juno data recorded in comparable conditions, within the constraints set by the Juno orbits (i.e. an apojove at $\sim 113 R_{J}$, cf. Figure 1a), we selected 120 intervals of 4 jovian rotation periods each $(\sim 40$ hours) over the portions of Juno's orbits located within $\pm 4^{\circ}$ of Jovicentric latitude and farther than $30 R_{J}$ from the planet (these limits are displayed in Figure 1a). Together, these intervals account for $\sim 13.5 \%$ of the total time spent by Juno within these limits (see Figure 1b).

Figure A1a,b show dynamic spectra of Juno/Waves survey data (Kurth \& Piker, 2019, NASA/PDS v1) on 2016/08/27 (around perijove 1) and 2016/10/16 (at $\sim 45 R_{J}$ from Jupiter and a latitude $\sim 9^{\circ}$ ), with intensities in $V^{2} / \mathrm{m}^{2} / \mathrm{Hz}$. The y-axis is labelled in channel number, which is a convenient display as the distribution of frequencies is logarithmic below $3 \mathrm{MHz}$ (i.e. below channel \#87, across the LFR-low, LFR-high and HFRlow receivers) and linear in the HFR-high receiver (channels \#88 and higher). Receiver limits are well visible: LFR-low band goes here from channel \#16 to \#42 (1 to $20 \mathrm{kHz}$ ), LFR-high from \#43 to \#60 (20 to $140 \mathrm{kHz}$ ), HFR-low from \#62 to \#87 (140 kHz to $3 \mathrm{MHz}$ ), and HFR-high from \#88 to \#125 (3 to $41 \mathrm{MHz}$ ). Jovian radio emissions are all the structured patches except the constant interference in HFR-high and HFR-low and the intense variable one in HFR-low in Figure A1a.

Figure A1c displays the apparent median flux density spectrum derived from "PDS v1" data, that includes normalization to $1 \mathrm{AU}$ (see below), for each of the 120 selected intervals. Again the band limits are clear, with large intensity jumps across them (up to $\geq 30 \mathrm{~dB}$ at edges of the HFR-low band, which in addition displays a much larger signal variability). The interference lines in HFR-high are very stable. Of course the intensity jumps are not physical, which shows the need for processing further the intensities.

\section{A1 Pre-processing of Juno/Waves data}

First, we checked if the Jovian radio signals at high frequency (DAM emission) significantly changed on the average between the Cassini and Juno eras, separated by $\geq$ 16 years. To do so, we used the Nancay Decameter Array catalog from Marques et al. 

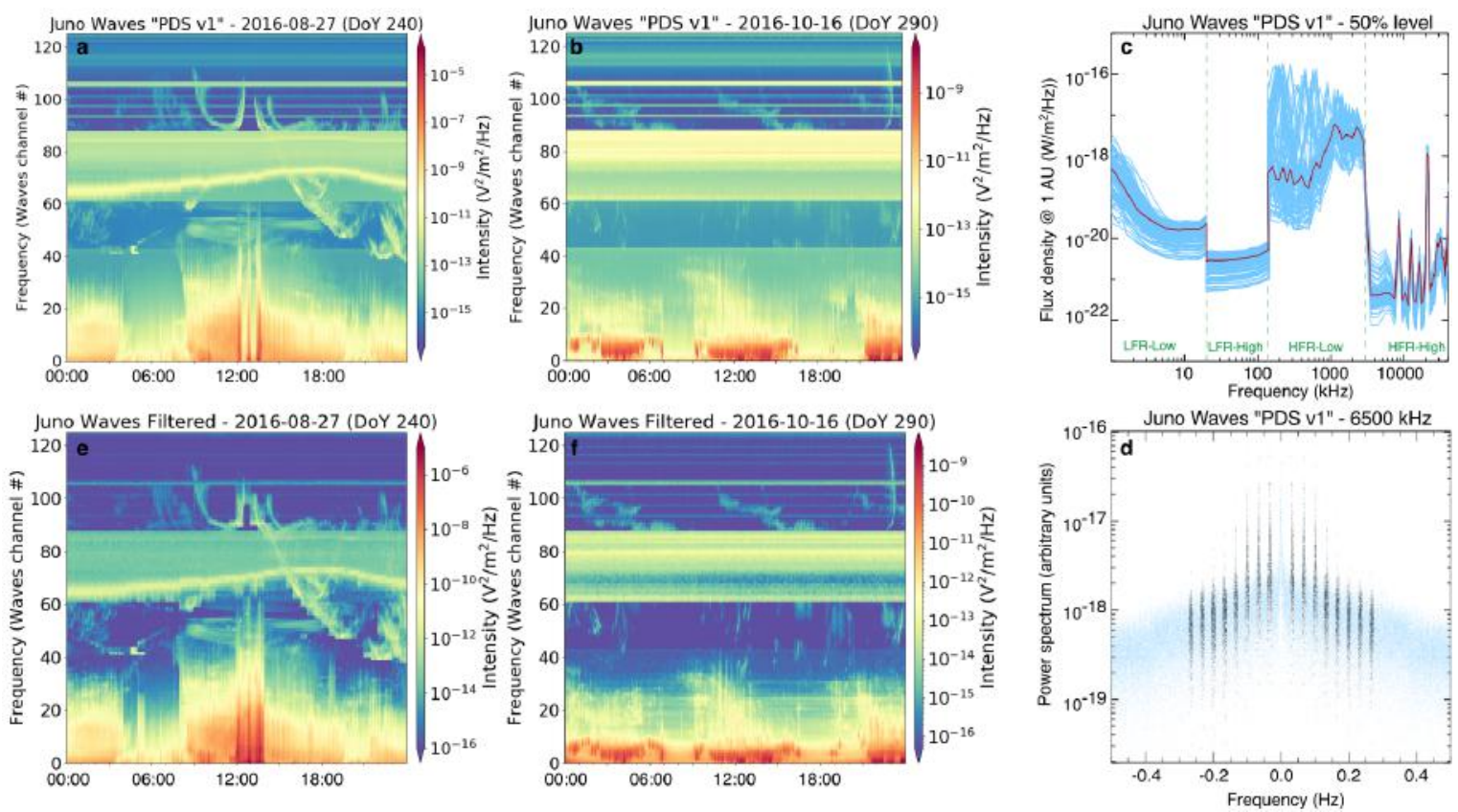

Figure A1. (a) Dynamic spectra of 24 h of Juno/Waves "PDS v1" survey data around perijove 1. Y-axis is in channel number, that combines log-spaced frequencies $<3 \mathrm{MHz}$ (channel \#87) and linearly-spaced frequencies above that frequency. Intensities are in $V^{2} / \mathrm{m}^{2} / \mathrm{Hz}$. Ephemeris information is listed along the X-axis. (b) Same as (a) for $24 \mathrm{~h}$ between perijoves 1 and 2, at 40 to $50 R_{J}$ from the planet. (c) Median flux density spectra derived from Juno/Waves PDS-v1 data over 120 selected intervals (in blue) and their overall median spectrum (in red), normalized to an observer's distance of 1 AU. Intensities have been converted to $W / m^{2} / H z$ by a simple division by the impedance of free space $\left(Z_{0}=377 \Omega\right.$ ). (d) Power spectrum of a time series of Juno/Waves measurements in channel $91(6.5 \mathrm{MHz})$. Blue parts of the power spectrum are set to 0 for filtering out interference not modulated by the spacecraft rotation and its harmonics (these are the intervals $N / 30 \pm 0.005 \mathrm{~Hz}$, with $N=1,8$, displayed in black). (e,f) Same as (a,b) after FFT-filtering (d).

(2017), extended since to the end of 2020, that encompasses the two epochs. Figure A2a shows the average intensity of each DAM event (the only Jovian radio component visible from Earth) from that catalog between late 1990 and 2020. Intensity values have been corrected for the variable Earth-Jupiter distance, also displayed on the Figure. Intensities appear very stable over the long term, with a $\sim 2 \mathrm{~dB}$ modulation at 11-12 year period (solar wind and Jovian year). The Cassini fly-by epoch, $\sim 2$ years before a solar activity maximum, is displayed with red symbols. The Juno tour, which started after the next solar activity maximum, is displayed with blue symbols. To better quantify the measured intensities, we display in Figure A2b the histograms of the intensity distributions of DAM events over the Cassini (red) and Juno (blue) eras, marking also their mean and median values (vertical solid and dashed lines). Figure A2c is similar to Figure A2b except that the blue (Juno) distribution is restricted to the 120 intervals of $40 \mathrm{~h}$ duration each - selected in Juno data (see above). We can see that the DAM mean intensities are identical between the two epochs (within $\sim 0.1 \mathrm{~dB}$ i.e. $\sim 2 \%$ ), while the median values have not varied by more than $0.3 \mathrm{~dB}(\sim 7 \%)$. We will see that these errors are negligible with respect to the other ones affecting the processing procedure. 
NDA catalog 1990-2020
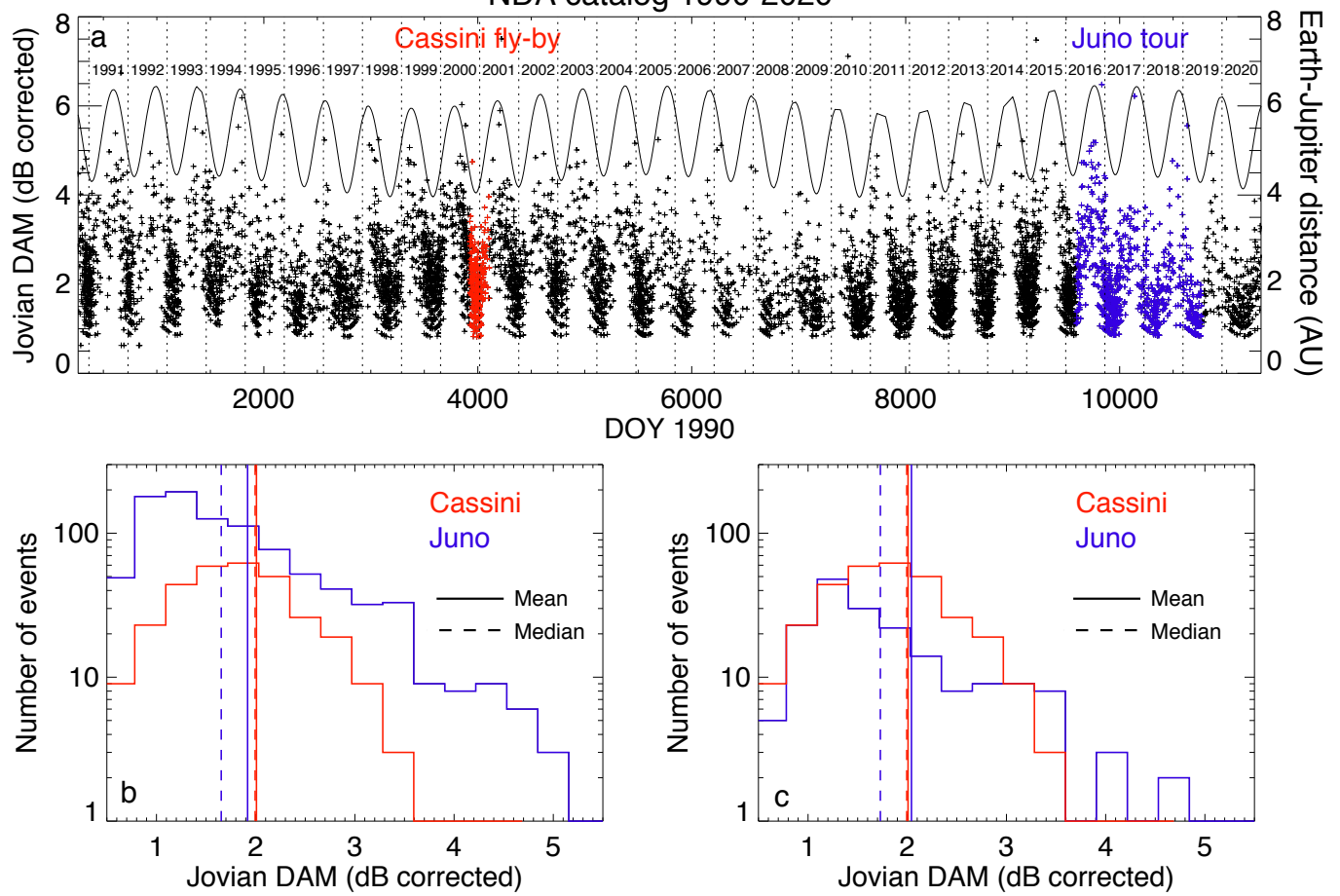

Figure A2. (a) DAM intensity from the Nançay Decameter Array catalog 1990-2020 (extended from Marques et al., 2017), averaged on each event of the catalog (of duration between a few minutes and 5 hours). Intensity values have been corrected for the variable Earth-Jupiter distance, also displayed in the figure. Measurements during the Cassini flyby are displayed in red, and those during the Juno tour (for the 3-year interval studied) in blue. (b) Histograms of DAM intensities measured with the NDA during the Cassini and Juno eras, with indication of the mean (solid line) and median (dashed line) of each histogram. (c) Same as (b) with the Juno era histogram computed only over the 120 intervals selected for our processing.

Second, we must pre-process the Juno/Waves data in order to obtain intensity values related to Jupiter signals only. In the case of Cassini data, it was enough to subtract the Galactic background (measured at times when Jupiter signal was absent and used for calibrating the Cassini data) and interpolate through a few discrete interference lines (Zarka et al., 2004). The interference and instrumental noise are much more severe on Juno. Thus we implemented a new filtering technique specifically adapted to Juno radio measurements.

Juno/Waves uses a single short dipole as an electric sensor (Kurth, Hospodarsky, et al., 2017). Such an antenna has a broad gain pattern with a minimum in the direction of the dipole, and a maximum perpendicular to it (in $\sin ^{2} \theta$ for an ideal short dipole, with $\theta$ the angle relative to the direction of the dipole; see e.g. Cecconi, 2010). As Juno rotates around an axis nearly perpendicular to this dipole, any signal entering via the antenna (except if coming exactly along the rotation axis) will be modulated at Juno's rotation period $P_{\text {Juno }}=30 \mathrm{~s}$, at $P_{\text {Juno }} / 2$ because an electric dipole is invariant by a rotation of $180^{\circ}$, and at higher harmonics $P_{\text {Juno }} / N$ because the antenna response is not purely sinusoidal (it is perturbed by the spacecraft body and its solar panels, and it depends on the incoming direction of the signal relative to the antenna, see Sampl et al., 2016). Indeed, when we compute the power spectrum of a time series of Juno/Waves mea- 
surements at any given frequency, it reveals many peaks at frequencies of $N / 30 \mathrm{~Hz}(N \geq$ 1), as displayed in Figure A1d for a typical frequency channel. Spacecraft- or instrumentgenerated interference are not modulated by the spacecraft rotation. Thus, in order to get rid of most non-Jupiter signals, we filtered out unmodulated signals.

In practice, for each Waves frequency channel and each window of $24 \mathrm{~h}$, we computed by FFT complex spectra of the corresponding times series, resampled at $1 \mathrm{~s}$ time resolution (to fulfilled the Nyquist-Shannon sampling criterion) with short gaps interpolated; then we set to 0 the resulting spectrum except for spectral ranges around the $N / 30 \mathrm{~Hz}$ frequencies, and performed an inverse FFT. Tests showed that an optimal result (in terms of interference removal and signal preservation) was obtained by retaining a window of $\pm 0.005 \mathrm{~Hz}$ (i.e. $\pm 15 \%$ of the fundamental frequency $1 / 30 \mathrm{~Hz}$ ) around harmonics with $N=1$ to 8 (out of a maximum of $N=15$ due to the initial data sampling at $1 \mathrm{sec}$, but harmonics 9 to 15 were found to contain only noise). Accordingly, only the black parts of Figure A1d are retained whereas light blue parts are set to 0. After FFT filtering, data are integrated in $15 \mathrm{~s}$ bins. This filtering results are illustrated on Figures A1e and A1f, that are the filtered versions of Figures A1a and A1b. A large part of interference is removed and the jumps between spectral bands are reduced, so that the Jovian radio emissions appear with a much better contrast.

Third, we compute and subtract a background at each frequency. In principle, FFT filtering should remove or considerably reduce any constant background. In practice, we see that a low-level background persists in bands LFR-low, LFR-high and HFR-high, and a relatively large one in HFR-low (that contains only noise and interference except close to perijoves). The background is computed at each frequency as the mode of the histogram (estimated via a robust mean) of a long series of measured values at that frequency. Figure A3a displays the background spectra computed per interval of 53 days between perijoves, as well as on the inbound trajectory prior to 2016/07/04. We see that except in the lower part of the HFR-low band, the background is very stable all along the tour. Thus we used a constant mean background for the entire 3-year interval studied. Figure A3b displays a typical time series at a clean frequency of the HFR-high band after FFT filtering. The periodic peaks correspond to apparent signal increases at perijoves due to decreasing distance. Figure A3c shows the same time series after background subtraction, that alters only low intensity values. The signal in panel A3c should consist exclusively of Jovian radio emissions. Then, normalization by $R^{2}$ is applied (with $R$ the distance between Juno and Jupiter's center) in order to derive the Jovian signal as observed from a distance of 1 AU. It is displayed in Figure A3d in flux density units $\left(\mathrm{W} / \mathrm{m}^{2} / \mathrm{Hz}\right.$, related to the processed signal in $V^{2} / \mathrm{m}^{2} / H z$ by a simple division by the impedance of free space, $Z_{0}=377 \Omega$ ). In spite of the error introduced, mostly near perijoves, by the fact that the radio sources are not at the center of Jupiter, the time series of Figure A3d shows a quasi constant peak level of Jovian radio emissions across the 3 -year interval studied, and periodic minima caused by the capability to detect weaker signals near perijoves, consistent with expectations.

The above pre-processing, consisting of FFT filtering, background subtraction, division by the impedance of free space $Z_{0}$ and normalization to $1 \mathrm{AU}$, was then applied to all data from the 120 intervals mentioned above.

\section{A2 Conversion of pre-processed Juno/Waves data into flux densities}

Following the method defined in Zarka et al. (2004), for each of the 120 selected intervals (lasting 4 Jovian rotation each), we computed from the pre-processed Juno/Waves data a $50 \%$ occurrence spectrum and a $1 \%$ occurrence spectrum (i.e. the levels at each frequency exceeded resp. $50 \%$ and $1 \%$ of the time). These spectra (in blue) and their overall medians are displayed in Figures A4a and A4b. In order to derive smooth conversion curves versus frequency, we computed polynomial fits of the median $50 \%$ (in red) 

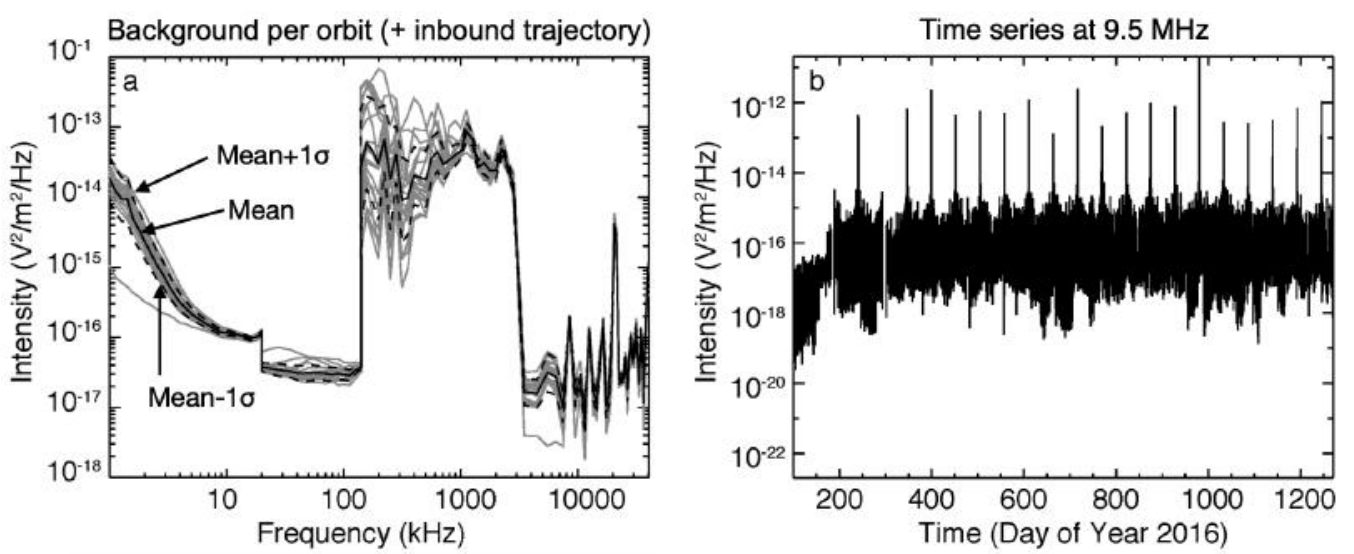

Time series at $9.5 \mathrm{MHz}$ - Background subtracted

Time series at $9.5 \mathrm{MHz}$ - background subtracted
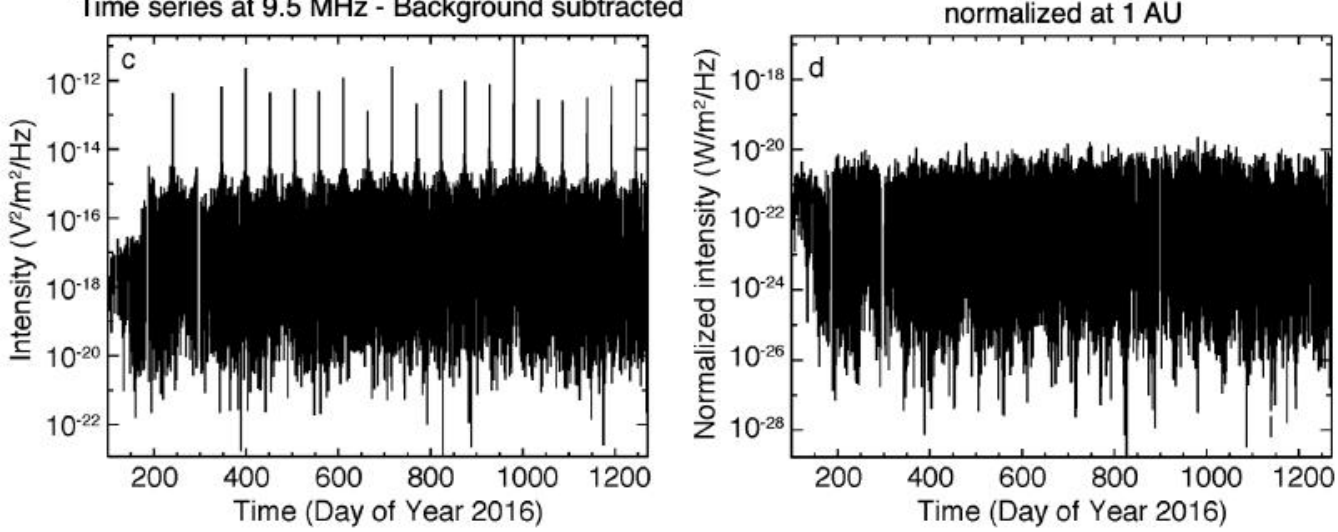

Figure A3. (a) Background spectra of Juno/Waves FFT-filtered survey measurements per Juno's orbit (+ during the inbound trajectory from 2016/04/09 to perijove 0 on 2016/07/05) are displayed in grey. The background with a lower low-frequency part is the one corresponding to the inbound trajectory, over which no Jovian trapped continuum is detected. The mean background is the black solid line, with its $1 \sigma$ variations limited by the dashed lines. (b) Time series of FFT-filtered intensities in channel 94. (c) Same as (b), after subtraction of mean background from (a). (d) Same as (c) after normalization to an observer's distance of 1 AU and conversion to flux densities.

and $1 \%$ (in black) Juno/Waves spectra of Figures A4a and A4b. A fit by polynomials of degree 5 was found to reproduce well the median spectra in the 3 lower bands. In the HFR-high band, the very stable residual interference must be taken into account in the conversion curve, thus a polynomial fit was not applicable. Instead, we only smoothed the median HFR-high spectra by a sliding 3-bin window. The resulting reference $50 \%$ and $1 \%$ spectra for Juno/Waves data are displayed in Figure A4c as solid red and black lines, respectively.

The reference spectra derived from Cassini data, that should be matched to the above reference Juno/Waves spectra, are displayed in Figure A4c as dashed lines. These spectra are the smoothed versions of the median $50 \%$ and $1 \%$ spectra obtained from the 21 intervals of Cassini observations of Jupiter studied in Zarka et al. (2004), and displayed in Figure 7a,b of that paper. As Cassini radio observations only covered the range $3.5 \mathrm{kHz}-$ 16.1 MHz, we used Voyager measurements of the Jovian radio spectrum from Zarka (1992) to extend the reference Cassini $50 \%$ and $1 \%$ spectra up to $40.5 \mathrm{MHz}$. The Voyager spec- 

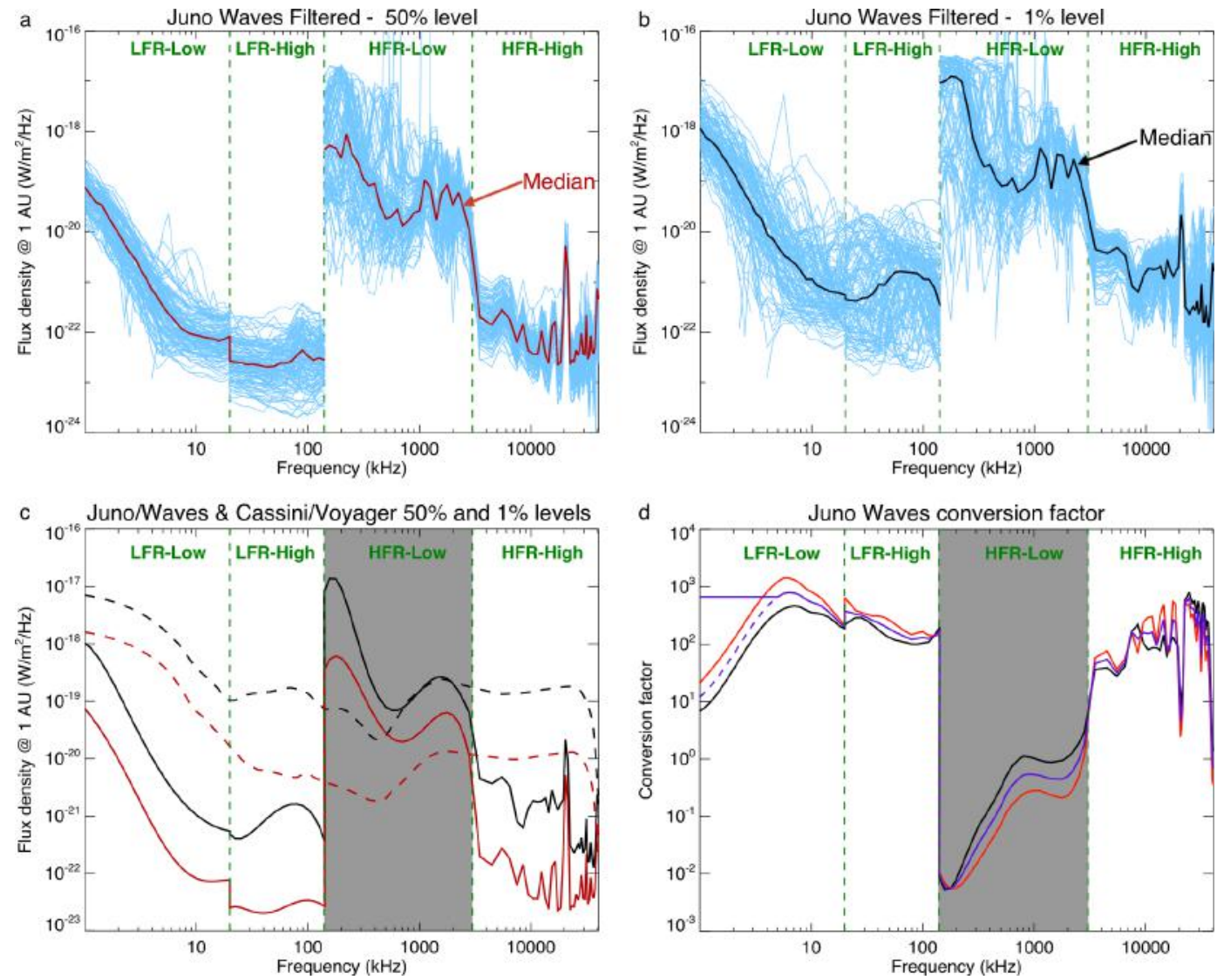

Figure A4. (a) Median flux density spectra computed over the 120 selected intervals of Juno/Waves data, after FFT-filtering, background subtraction and normalization to an observer's distance of $1 \mathrm{AU}$ (blue lines). The red line is the median over all individual median spectra. (b) same as (a), but for the first percentile spectra i.e. the flux density at each frequency exceeded by $1 \%$ of the measurements in each of the 120 intervals. The black line is the median over all individual $1 \%$ occurrence spectra. (c) Smoothed median spectra from panels (a) and (b) (solid lines) and Cassini-Voyager 50\% and 1\% spectra derived as explained in the caption of Figure $\mathrm{S} 1$ (dashed lines). The HFR-low band is grey-shaded because this procedure based on longterm statistics does not apply to it (see text). (d) Conversion curves deduced from the ratios of Cassini-Voyager to Juno 50\% (red) and 1\% (black) spectra from panel (c). The purple line is the geometrical average of the red and black curves. A constant value is used below $5 \mathrm{kHz}$ in replacement of the computed ones (dashed), to take into account the presence of trapped continuum (see text). 
trum matches well the Cassini $1 \%$ spectrum, and it was downscaled in intensity to also extend the $50 \%$ spectrum. Figure S1 in the Supporting Information provides some details on the construction of the Cassini-Voyager reference spectra. These spectra represent the true flux densities that should be statistically measured at $1 \mathrm{AU}$, at respectively the $1 \%$ and the $50 \%$ occurrence levels. We interpolated them on Juno/Waves frequencies. Finally, dividing frequency by frequency the black dashed curve by the black solid one, and the red dashed curve by the red solid one, provides two estimates of the Juno/Waves conversion curve, plotted in Figure A4d with their corresponding colors (the one resulting from the ratio of $1 \%$ spectra in black, and that deduced from $50 \%$ spectra in red). They are very close to each other over the entire spectral range of Juno/Waves. We computed their geometrical average (in purple), that will be used as the final conversion curve that will allow us to convert Juno/Waves FFT-filtered data into flux densities.

Taking into account the shift of each curve (black or red) to their geometrical average (purple), and the secondary sources of errors noted above (difference of actual emission levels at Cassini(-Voyager) and Juno epochs, the effect of the background subtraction on low intensity values, smoothing of the reference spectra), we estimate that an error of a factor $\leq 2$ should affect the estimated Juno/Waves flux densities in the LFRhigh and HFR-high bands, and in the LFR-low band above $5 \mathrm{kHz}$.

Below $5 \mathrm{kHz}$, measurements from Juno inside the Jovian magnetosphere and from Cassini which stayed outside of it (Kurth et al., 2002) differ due to the presence of the trapped continuum (Gurnett \& Scarf, 1983). This component does not originate close to the planet and its intensity is not expected to vary in $1 / R^{2}$. Its presence explains that the intensities measured by Juno below $5 \mathrm{kHz}$ are $2-3$ orders of magnitude higher than those above $10 \mathrm{kHz}$, whereas this ratio is only $1-2$ orders of magnitude for Cassini. This explains in turn the steep decrease of our conversion curve in Figure A4d, that is invalid below $5 \mathrm{kHz}$. One way to possibly solve this problem would be to use measurements from a secondary calibrator, i.e. a spacecraft having performed radio measurements both inside and outside Jupiter's magnetosphere, such as Voyager or Galileo. Measurements from that spacecraft outside the magnetosphere can be calibrated against Cassini measurements (themselves calibrated on the Galactic radio background), and they can then be compared to Juno/Waves measurements inside the magnetosphere. Such a complex process is beyond the scope of the present paper, as the range below $5 \mathrm{kHz}$ only concerns the flux densities (not the occurrences) at the lower end of the nLF, QP and bKOM components. For simplicity, we simply propose to use below $5 \mathrm{kHz}$ a constant conversion factor that is the one computed at $5 \mathrm{kHz}$. The purple curve of Figure A4d has been adapted accordingly.

The situation is even more complex in the HFR-low band. The purple curve is several magnitude orders lower there than in the other bands. The reason is that due to the very low sensitivity of the HFR-low band, Jovian signals are only detected for a few hours around the perijoves (FFT of HFR-low data time series show no peak at $N / 30 \mathrm{~Hz}$ outside of perijoves). Therefore, the $1 \%$ and $50 \%$ levels computed from the 120 selected intervals, all located beyond $30 R_{J}$ from the planet, actually characterize only the noise and not the Jovian radio spectrum. The above procedure is therefore not relevant for estimating the flux density in the HFR-low band. This is why this band is shaded in Panels A4c,d. We have adapted the procedure to the specificity of this band as follows.

We considered only 19 intervals of $4 \mathrm{~h}$, i.e. $\pm 2 \mathrm{~h}$, around perijoves \#1 to \#20 (instruments were off during perijove \#2), as these are the only intervals during which Jovian signals were recorded in HFR-low. During these intervals, FFT filtering was performed on $30 \mathrm{~min}$. windows (instead of $24 \mathrm{~h}$ for the other bands) to minimize the strong signal variations due to the rapidly changing distance from the spacecraft to the radio sources. Only the $1 \%$ occurrence level was computed at each frequency, because the $50 \%$ level is strongly polluted by the residual intense frequency-variable interference that appears in this band near perijoves (see Figures A1a,e). 

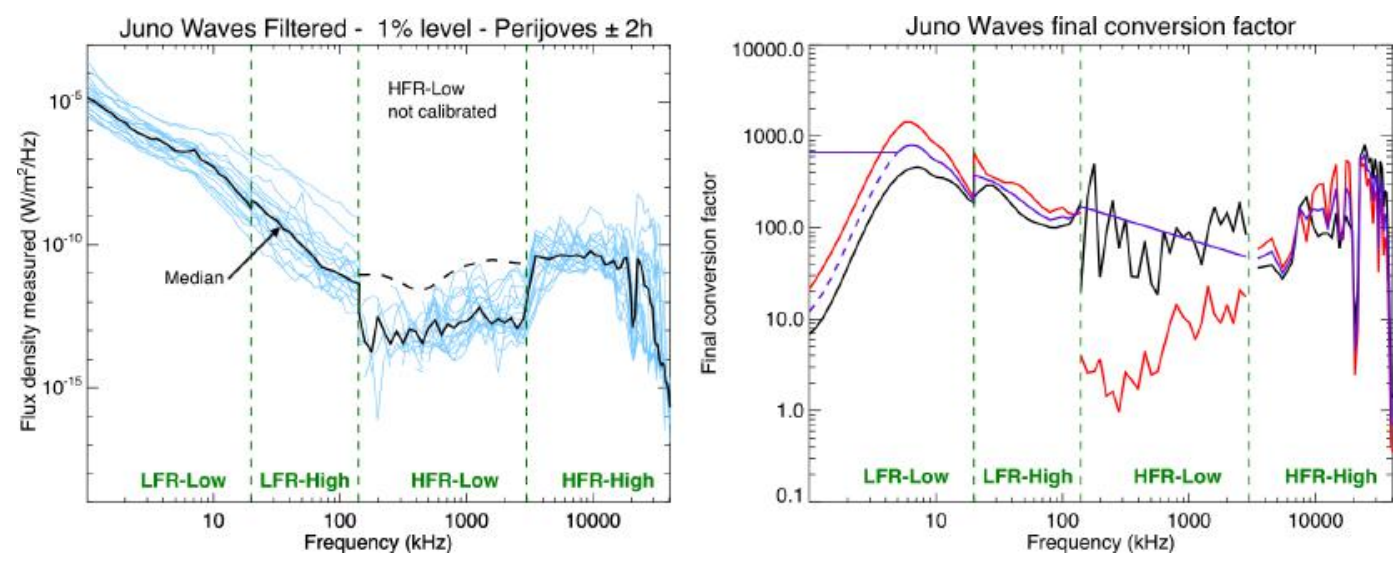

Figure A5. Same as Figure A4 but focused on the perijoves, for estimating the flux density of the HFR-low band.(a) First percentile spectra i.e. the flux density at each frequency exceeded by $1 \%$ of the measurements in each of the 19 intervals around perijoves. The black line is the median over all individual $1 \%$ occurrence spectra. Measurements have been processed and converted to flux densities in LFR-low, LFR-high and HFR-high bands, and pre-processed only (FFT-filtered, background subtracted) in the HFR-low band. The dashed curve is the portion of the Cassini 1\% reference spectrum (from Figure A4c), up-scaled to match the level of the signals on both sides of the HFR-low band. (b) Same as Figure A4d, completed by the conversion curve in the HFR-low band. The black line is deduced from panel (a). The red line is deduced from $50 \%$ spectra, which are too much polluted to be used for our processing procedure. The purple line is the linear interpolation of the black line that matches the conversion curve at both edges of the HFR-low band.

Figure A5a displays the $1 \%$ occurrence spectra measured by Juno/Waves in the above 19 intervals (blue lines), and their median (black solid line). In the LFR-low, LFRhigh and HFR-high bands, the spectra are processed using the curve of Figure A4d (but not normalized to a fixed distance), while they are only pre-processed (FFT filtered and background-subtracted) in the HFR-low band. The dashed curve in the HFR-low band is the portion of the Cassini 1\% reference spectrum (from Figure A4c), upscaled due to the much lower distance to the source in order to match the level of the processed signals at the upper end of the LFR-high and at the lower end of the HFR-high bands. This dashed curve is used as the true flux densities that should be measured by Juno/Waves in the HFR-low band, assuming that the Jovian radio spectrum is similar as seen from the high latitudes sampled around the perijoves and from the low latitudes sampled by Cassini. This assumption has no reason to be correct. It is only the simplest one that we can make, and it matches well the estimated levels on both sides of the band. But note that we will not derive a true reliable conversion curve in the HFR-low band, but only a reasonable estimate. Similar to the other bands, this estimated conversion curve is computed as the ratio of the Cassini $1 \%$ spectrum (dashed) to the Juno/Waves one (solid). It is displayed in Figure A5b as the black solid line in the HFR-low band, together with the conversion curves of the other bands reproduced from Figure A4d.

The red line in the HFR-low band is the conversion curve that would be deduced from the ratio of the Cassini $50 \%$ spectrum to the Juno/Waves one (not shown in Figure A5a). It is only displayed here for information, and will not be used, because the Juno/Waves $50 \%$ spectrum in the HFR-low band is strongly overestimated due to the contribution of the residual frequency-variable interference, and consequently the conversion curve is largely underestimated. We will thus use only the HFR-low conversion curve deduced 
from the ratio of the $1 \%$ spectra (solid black line). It is very noisy, due to the low statistics available in the nineteen $4 \mathrm{~h}$ intervals used, and possibly due to residual interference. In order to obtain a smooth conversion curve, we noted that an acceptable fit to this curve is simply the straight line connecting conversion curves outside the HFR-low band (solid purple line). We adopted that solution to complete the Juno/Waves conversion curve.

The error on the flux densities thus derived in the HFR-low band is difficult to estimate, due to the assumptions made. They are likely larger than in the other bands, but also likely much less than an order of magnitude, considering the consistency of the solutions across the various bands and the fluctuations of the black line around the purple one in Figure A5b (in the HFR-low band). Although not perfect, this is a significant improvement over PDS data, that show intensity jumps between bands (Figure A1c), contain interference and background, and are not directly related to flux densities.
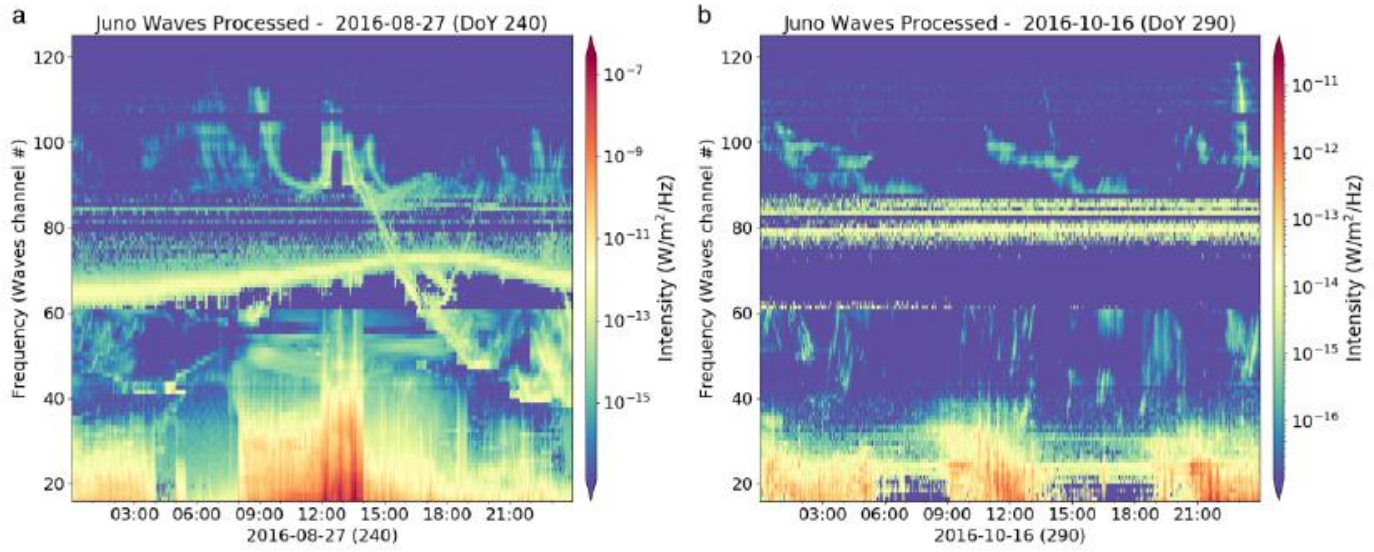

Figure A6. Processed versions derived from Figures A1a,b and then A1e,f by application of the conversion curve of Figure A5b.

Finally, the following steps have to be performed to process the Juno/Waves data and convert them to flux densities:

- resample the data (provided by the NASA/PDS, in linear scale of $V^{2} / m^{2} / H z$ ) to $1 \mathrm{~s}$ and interpolate short gaps;

- apply an FFT filtering (the window size is not crucial; $24 \mathrm{~h}$ is generally suitable except close to perijoves, where a shorter window is needed; we used $30 \mathrm{~min}$ in this study within $\pm 2 \mathrm{~h}$ of the perijoves);

- optionally subtract a background spectrum;

- multiply by the adequate conversion coefficients purple line of Figure A5b for converting "PDS v1" data). Flux densities in $W / m^{2} / H z$ are then obtained by dividing the results by the free space impedance $Z_{0}=377 \Omega$;

- optionally, correct for the $1 / R^{2}$ dependence to normalize the flux densities to a constant distance.

Figures A6a,b are the processed versions of Figures A1a,b. The displayed intensities have now a physical meaning. Most of the interference is removed (except in the HFR-low band), the jumps between spectral bands are further reduced to become quasinegligible, and the Jovian radio emissions show up clearly with a good contrast over a near-zero background. 
Note that our flux density conversion can be extended to burst-mode data by comparison with simultaneous survey-mode data used as a reference.

\section{Data Availability Statement}

The Juno/Waves "PDS v1" data used in this manuscript in input of our processing pipeline are available from the Planetary Data System at https://doi.org/10.17189/ 1519710 (Kurth \& Piker, 2019). The processed data converted to flux densities can be accessed at https://doi.org/10.25935/6jg4-mk86 (Louis, Zarka, \& Cecconi, 2021), along with tables of conversion factors and background values in each frequency channel. All the ExPRES simulation data are available at https://doi.org/10.25935/kpge -zb59 (Louis, Cecconi, \& Loh, 2020). The Juno/Waves catalogue of Section 4 is available at https://doi.org/10.25935/nhb2-wy29 (Louis, Zarka, Cecconi, \& Kurth, 2021)

\section{Acknowledgments}

The authors acknowledge the support from W. Kurth and the Juno/Waves team, as well as valuable comments from W. Kurth on the manuscript. C.L. acknowledges funding from the CNES, and thanks A. Loh for fruitful discussions. Co-authors from LESIA acknowledge fundings support from the CNES, PNP, PNST and Plas@Par.

\section{References}

Alexander, J. K., Carr, T. D., Thieman, J. R., Schauble, J. J., \& Riddle, A. C. (1981, September). Synoptic observations of Jupiter's radio emissions: average statistical properties observed by Voyager. Journal of Geophysical Research, 86(A10), 8529-8545. doi: 10.1029/JA086iA10p08529

Aubier, A., Boudjada, M. Y., Moreau, P., Galopeau, P. H. M., Lecacheux, A., \& Rucker, H. O. (2000, February). Statistical studies of jovian decameter emissions observed during the same period by Nancay Decameter Array (France) and WAVES experiment aboard Wind spacecraft. Astronomy and Astrophysics, 354, 1101-1109.

Barbosa, D. D. (1982, May). Low-level VLF and LR radio emissions observed at earth and Jupiter. Reviews of Geophysics and Space Physics, 20, 316-334. doi: 10.1029/RG020i002p00316

Bigg, E. K. (1964, September). Influence of the Satellite Io on Jupiter's Decametric Emission. Nature, 203, 1008-1010. doi: 10.1038/2031008a0

Boischot, A., \& Aubier, M. G. (1981, September). The Jovian decametric arcs as an interference pattern. Journal of Geophysical Research, 86, 8561-8563. doi: 10 .1029/JA086iA10p08561

Boischot, A., Lecacheux, A., Kaiser, M. L., Desch, M. D., Alexander, J. K., \& Warwick, J. W. (1981, September). Radio Jupiter after Voyager: An overview of the planetary radio astronomy observations. Journal of Geophysical Research (Space Physics), 86(A10), 8213-8226. doi: 10.1029/JA086iA10p08213

Carr, T. D., Desch, M. D., \& Alexander, J. K. (1983). Phenomenology of magnetospheric radio emissions. In A. J. Dessler (Ed.), Physics of the jovian magnetosphere (p. 226-284).

Cecconi, B. (2010, January). Goniopolarimetric techniques for low-frequency radio astronomy in space. ISSI Scientific Reports Series, 9, 263-277.

Connerney, J. E. P., Kotsiaros, S., Oliversen, R. J., Espley, J. R., Joergensen, J. L., Joergensen, P. S., .. Levin, S. M. (2018, March). A New Model of Jupiter's Magnetic Field From Juno's First Nine Orbits. Geophysical Research Letters, 45, 2590-2596. doi: 10.1002/2018GL077312

Desch, M. D. (1994, February). Jupiter Radio Bursts and Particle Acceleration. Astrophysical Journal Supplement, 90, 541. doi: 10.1086/191872 
Divine, N., \& Garrett, H. B. （1983, September). Charged particle distributions in Jupiter's magnetosphere. Journal of Geophysical Research (Space Physics), 88(A9), 6889-6903. doi: 10.1029/JA088iA09p06889

Dulk, G. A., Erickson, W. C., Manning, R., \& Bougeret, J. L.

(2001, January). Calibration of low-frequency radio telescopes using the galactic background radiation. Astronomy and Astrophysics, 365, 294-300. doi: 10.1051/0004-6361:20000006

Galopeau, P. H. M., \& Boudjada, M. Y. (2016, April). An oblate beaming cone for Io-controlled Jovian decameter emission. Journal of Geophysical Research (Space Physics), 121, 3120-3138. doi: 10.1002/2015JA021038

Gurnett, D. A., Kurth, W. S., Kirchner, D. L., Hospodarsky, G. B., Averkamp, T. F., Zarka, P., ... Pedersen, A. (2004, September). The Cassini Radio and Plasma Wave Investigation. $\quad$ Space Science Reviews, 114, 395-463. doi: 10.1007/s11214-004-1434-0

Gurnett, D. A., Kurth, W. S., \& Scarf, F. L. (1983, Mar). Narrowband electromagnetic emissions from Jupiter's magnetosphere. Nature, 302(5907), 385-388. doi: $10.1038 / 302385 \mathrm{a} 0$

Gurnett, D. A., Kurth, W. S., Shaw, R. R., Roux, A., Gendrin, R., Kennel, C. F., ... Shawhan, S. D. (1992, May). The Galileo Plasma wave investigation. Space Science Reviews, 60(1-4), 341-355. doi: 10.1007/BF00216861

Gurnett, D. A., \& Scarf, F. L. (1983). Physics of the Jovian magnetosphere. 8. Plasma waves in the Jovian magnetosphere. In Physics of the jovian magnetosphere (p. 285-316).

Hess, S., Cecconi, B., \& Zarka, P. (2008, July). Modeling of Io-Jupiter decameter arcs, emission beaming and energy source. Geophysical Research Letters, 35, L13107. doi: 10.1029/2008GL033656

Higgins, C. A., Menietti, J. D., \& Christopher, I. W. (2006, July). Europa control of Jovian radio emission: A Galileo study. Geophysical Research Letters, 33 L14110. doi: 10.1029/2006GL026218

Hill, T. W., Dessler, A. J., \& Michel, F. C. (1974). Configuration of the Jovian magnetosphere. Geophysical Research Letters, 1, 3-6. doi: 10.1029/ GL001i001p00003

Hospodarsky, G. B., Kurth, W. S., Cecconi, B., Gurnett, D. A., Kaiser, M. L., Desch, M. D., \& Zarka, P. (2004, Sep). Simultaneous observations of Jovian quasi-periodic radio emissions by the Galileo and Cassini spacecraft. Journal of Geophysical Research (Space Physics), 109(A9), A09S07. doi: 10.1029/2003JA010263

Imai, M., Greathouse, T. K., Kurth, W. S., Gladstone, G. R., Louis, C. K., Zarka, P., ... Connerney, J. E. P. (2019, Jan). Probing Jovian Broadband Kilometric Radio Sources Tied to the Ultraviolet Main Auroral Oval With Juno. Geophysical Research Letters, 46(2), 571-579. doi: 10.1029/2018GL081227

Imai, M., Imai, K., Higgins, C. A., \& Thieman, J. R. (2011, December). Comparison between Cassini and Voyager observations of Jupiter's decametric and hectometric radio emissions. Journal of Geophysical Research (Space Physics), 116(A12), A12233. doi: 10.1029/2011JA016456

Imai, M., Kurth, W. S., Hospodarsky, G. B., Bolton, S. J., Connerney, J. E. P., \& Levin, S. M. (2017, July). Direction-finding measurements of Jovian lowfrequency radio components by Juno near Perijove $1 . \quad$ Geophysical Research Letters, 44, 6508-6516. doi: 10.1002/2017GL072850

Jones, D. (1988, January). Planetary radio emissions from low magnetic latitudes Observations and theories. In Planetary radio emissions ii (p. 245-281).

Kaiser, M. L. (1993, October). Time-variable magnetospheric radio emissions from Jupiter. Journal of Geophysical Research, 98(E10), 18757-18766. doi: 10.1029/ 93JE01279

Kaiser, M. L., \& Desch, M. D. (1980, May). Narrow-band Jovian kilometric ra- 
diation: A new radio component. Geophysical Research Letters, 7(5), 389-392. doi: 10.1029/GL007i005p00389

Kaiser, M. L., Desch, M. D., Farrell, W. M., MacDowall, R. J., Stone, R. G., Lecacheux, A., ... Zarka, P. (1992, April). Ulysses observations of escaping VLF emissions from Jupiter. Geophysical Research Letters, 19(7), 649-652. doi: 10.1029/92GL00387

Kimura, T., Cecconi, B., Zarka, P., Kasaba, Y., Tsuchiya, F., Misawa, H., \& Morioka, A. (2012, November). Polarization and direction of arrival of Jovian quasiperiodic bursts observed by Cassini. Journal of Geophysical Research (Space Physics), 117(A11), A11209. doi: 10.1029/2012JA017506

Kimura, T., Tsuchiya, F., Misawa, H., Morioka, A., \& Nozawa, H. (2008a, June). Occurrence and source characteristics of the high-latitude components of Jovian broadband kilometric radiation. Planetary and Space Science, 56(8), 1155-1168. doi: 10.1016/j.pss.2008.03.001

Kimura, T., Tsuchiya, F., Misawa, H., Morioka, A., \& Nozawa, H. (2008b, December). Radiation characteristics of quasi-periodic radio bursts in the Jovian high-latitude region. Planetary and Space Science, 56(15), 1967-1976. doi: 10.1016/j.pss.2008.09.021

Kurth, W. S., Gurnett, D. A., Hospodarsky, G. B., Farrell, W. M., Roux, A., Dougherty, M. K., ... Alexander, C. J. (2002, February). The dusk flank of Jupiter's magnetosphere. Nature, 415(6875), 991-994. doi: 10.1038/415991a

Kurth, W. S., Gurnett, D. A., \& Scarf, F. L. (1980). Spatial and temporal studies of jovian kilometric radiation. Geophysical Research Letters, 7(1), 61-64. Retrieved from https://agupubs.onlinelibrary.wiley.com/doi/abs/ 10.1029/GL007i001p00061 doi: https://doi.org/10.1029/GL007i001p00061

Kurth, W. S., Gurnett, D. A., \& Scarf, F. L. (1989, Jun). Jovian Type III radio bursts. Journal of Geophysical Research,94(A6), 6917-6924. doi: 10.1029/ JA094iA06p06917

Kurth, W. S., Hospodarsky, G. B., Kirchner, D. L., Mokrzycki, B. T., Averkamp, T. F., Robison, W. T., ... Zarka, P. (2017, November). The Juno Waves Investigation. $\quad$ Space Science Reviews, 213, 347-392. doi: 10.1007/ s11214-017-0396-y

Kurth, W. S., Imai, M., Hospodarsky, G. B., Gurnett, D. A., Louarn, P., Valek, P., ... Zarka, P. (2017, July). A new view of Jupiter's auroral radio spectrum. Geophysical Research Letters, 44, 7114-7121. doi: 10.1002/2017GL072889

Kurth, W. S., \& Piker, C. W. (2019). Juno E/J/S/SS Waves Calibrated Survey Full Resolution V1.0, NO-E/J/SS-WAV-3-CDR-SRVFULL-V1.0. NASA Planetary Data System. doi: 10.17189/1519710

Ladreiter, H. P., Zarka, P., \& Lacacheux, A. (1994, November). Direction finding study of Jovian hectometric and broadband kilometric radio emissions: Evidence for their auroral origin. Planetary Space Science, 42, 919-931. doi: 10.1016/0032-0633(94)90052-3

Lamy, L., Zarka, P., Cecconi, B., Klein, L., Masson, S., Denis, L., ... Viou, C. (2017, January). 1977-2017: 40 years of decametric observations of Jupiter and the Sun with the Nancay Decameter Array. In G. Fischer, G. Mann, M. Panchenko, \& P. Zarka (Eds.), Planetary radio emissions viii (p. 455-466). doi: $10.1553 /$ PRE8s455

Lecacheux, A., Pedersen, B. M., Zarka, P., Aubier, M. G., Desch, M. D., Farrell, W. M., ... Stone, R. G. (1992, June). In ecliptic observations of Jovian radio emissions by Ulysses comparison with Voyager results. Geophysical Research Letters, 19(12), 1307-1310. doi: 10.1029/92GL01037

Louarn, P., Allegrini, F., McComas, D. J., Valek, P. W., Kurth, W. S., André, N., ... Zink, J. L. (2017, May). Generation of the Jovian hectometric radiation: First lessons from Juno. Geophysical Research Letters, 44, 4439-4446. doi: 10.1002/2017GL072923 
Louarn, P., Allegrini, F., McComas, D. J., Valek, P. W., Kurth, W. S., André, N., ... Wilson, R. J. (2018, September). Observation of Electron Conics by Juno: Implications for Radio Generation and Acceleration Processes. Geophysical Research Letters, 45(18), 9408-9416. doi: 10.1029/2018GL078973

Louis, C. K., Cecconi, B., \& Loh, A. (2020). ExPRES Jovian Radio Emission Simulations Data Collection (Version 01). PADC. doi: 10.25935/KPGE-ZB59

Louis, C. K., Hess, S. L. G., Cecconi, B., Zarka, P., Lamy, L., Aicardi, S., \& Loh, A. $\quad(2019$, Jul). $\quad$ ExPRES: an Exoplanetary and Planetary Radio Emissions Simulator. Astronomy and Astrophysics, 627, A30. doi: 10.1051/0004-6361/201935161

Louis, C. K., Lamy, L., Zarka, P., Cecconi, B., \& Hess, S. L. G. (2017, September). Detection of Jupiter decametric emissions controlled by Europa and Ganymede with Voyager/PRA and Cassini/RPWS. Journal of Geophysical Research (Space Physics), 122, 9228-9247. doi: 10.1002/2016JA023779

Louis, C. K., Lamy, L., Zarka, P., Cecconi, B., Imai, M., Kurth, W. S., ... Levin, S. M. (2017, September). Io-Jupiter decametric arcs observed by Juno/Waves compared to ExPRES simulations. Geophysical Research Letters, 44, 92259232. doi: 10.1002/2017GL073036

Louis, C. K., Louarn, P., Allegrini, F., Kurth, W. S., \& Szalay, J. R. $\quad$ (2020, October). Ganymede-Induced Decametric Radio Emission: In Situ Observations and Measurements by Juno. Geophysical Research Letters, 47(20), e90021. doi: 10.1029/2020GL090021

Louis, C. K., Prangé, R., Lamy, L., Zarka, P., Imai, M., Kurth, W. S., \& Connerney, J. E. P. (2019, November). Jovian Auroral Radio Sources Detected In Situ by Juno/Waves: Comparisons With Model Auroral Ovals and Simultaneous HST FUV Images. Geophysical Research Letters, 46(21), 11,606-11,614. doi: 10.1029/2019GL084799

Louis, C. K., Zarka, P., \& Cecconi, B. (2021). Juno/Waves estimated flux density Collection (Version 1.0). PADC/MASER. doi: 10.25935/6jg4-mk86

Louis, C. K., Zarka, P., Cecconi, B., \& Kurth, W. S. (2021). Catalogue of Jupiter radio emissions identified in the Juno/Waves observations (Version 1.0). PADC/MASER. doi: 10.25935/nhb2-wy29

MacDowall, R. J., Kaiser, M. L., Desch, M. D., Farrell, W. M., Hess, R. A., \& Stone, R. G. (1993, Nov). Quasiperiodic Jovian Radio bursts: observations from the Ulysses Radio and Plasma Wave Experiment. Planetary and Space Science, 41(11-12), 1059-1072. doi: 10.1016/0032-0633(93)90109-F

Manning, R., \& Dulk, G. A. (2001, June). The Galactic background radiation from 0.2 to $13.8 \mathrm{MHz}$. Astronomy and Astrophysics, 372, 663-666. doi: 10.1051/ 0004-6361:20010516

Marques, M. S., Zarka, P., Echer, E., Ryabov, V. B., Alves, M. V., Denis, L., \& Coffre, A. (2017, July). Statistical analysis of $26 \mathrm{yr}$ of observations of decametric radio emissions from Jupiter. Astronomy \& Astrophysics, 604, A17. doi: 10.1051/0004-6361/201630025

Menietti, J. D., Gurnett, D. A., Kurth, W. S., \& Groene, J. B. （1998, December). Control of Jovian radio emission by Ganymede. Geophysical Research Letters, 25, 4281-4284. doi: 10.1029/1998GL900112

Nakamura, Y., Kasaba, Y., Kimura, T., Lamy, L., Cecconi, B., Fischer, G., ... Morioka, A. (2019, November). Seasonal variation of north-south asymmetry in the intensity of Saturn Kilometric Radiation from 2004 to $2017 . \quad$ Planetary and Space Science, 178, 104711. doi: 10.1016/j.pss.2019.104711

Reiner, M. J., Fainberg, J., Stone, R. G., Kaiser, M. L., Desch, M. D., Manning, R., ... Pedersen, B. M. (1993, Jul). Source characteristics of Jovian narrowband kilometric radio emissions. Journal of Geophysical Research, 98(E7), 13163-15000. doi: 10.1029/93JE00536

Ronnmark, K. (1992, January). Conversion of Upper Hybrid waves into magneto- 
spheric radiation. In Planetary radio emissions iii (p. 405-417).

Sampl, M., Macher, W., Oswald, T., Plettemeier, D., Rucker, H. O., \& Kurth, W. S. (2016). Juno model rheometry and simulation. Radio Science, 51(10), 16271635. Retrieved from https://agupubs.onlinelibrary.wiley.com/doi/abs/ 10.1002/2016RS005954 doi: https://doi.org/10.1002/2016RS005954

Steinberg, J. L., Lacombe, C., Zarka, P., Hoang, S., \& Perche, C. $\quad$ (2004, June). Terrestrial low-frequency bursts: Escape paths of radio waves through the bow shock. Planetary and Space Science, 52(7), 643-660. doi: 10.1016/ j.pss.2003.12.005

Stone, R. G., Pedersen, B. M., Harvey, C. C., Canu, P., Cornilleau-Wehrlin, N., Desch, M. D., ... Zarka, P. (1992, September). Ulysses Radio and Plasma Wave Observations in the Jupiter Environment. Science, 257(5076), 1524-1531. doi: 10.1126/science.257.5076.1524

Treumann, R. A. (2006, August). The electron-cyclotron maser for astrophysical application. Astronomy \&3 Astrophysicsr, 13, 229-315. doi: 10.1007/s00159-006 $-0001-y$

Turner, J. D., Zarka, P., Grießmeier, J.-M., Lazio, J., Cecconi, B., Emilio Enriquez, J., .. de Pater, I. (2021, January). The search for radio emission from the exoplanetary systems 55 Cancri, $v$ Andromedae, and $\tau$ Boötis using LOFAR beam-formed observations. Astronomy and Astrophysics, 645, A59. doi: 10.1051/0004-6361/201937201

Zarka, P. (1992, Aug). The auroral radio emissions from planetary magnetospheres: What do we know, what don't we know, what do we learn from them? $A d$ vances in Space Research, 12(8), 99-115. doi: 10.1016/0273-1177(92)90383-9

Zarka, P. (1998, September). Auroral radio emissions at the outer planets: Observations and theories. Journal of Geophysics Research, 103, 20159-20194. doi: 10 $.1029 / 98 J E 01323$

Zarka, P. (2002). Magnetospheres: Jupiter, Radio Emissions. In P. Murdin (Ed.), Encyclopedia of astronomy and astrophysics (p. E2329). doi $10.1888 / 0333750888 / 2329$

Zarka, P. (2004, January). Radio and plasma waves at the outer planets. Advances in Space Research, 33, 2045-2060. doi: 10.1016/j.asr.2003.07.055

Zarka, P. (2007, April). Plasma interactions of exoplanets with their parent star and associated radio emissions. Planetary Space Science, 55, 598-617. doi: 10 $.1016 /$ j.pss.2006.05.045

Zarka, P., Cecconi, B., \& Kurth, W. S. (2004, September). Jupiter's low-frequency radio spectrum from Cassini/Radio and Plasma Wave Science (RPWS) absolute flux density measurements. Journal of Geophysical Research (Space Physics), 109, A09S15. doi: 10.1029/2003JA010260

Zarka, P., Marques, M. S., Louis, C., Ryabov, V. B., Lamy, L., Echer, E., \& Cecconi, B. (2018, October). Jupiter radio emission induced by Ganymede and consequences for the radio detection of exoplanets. Astronomy and Astrophysics, 618, A84. doi: 10.1051/0004-6361/201833586

Zarka, P., Queinnec, J., \& Crary, F. J. (2001, August). Low-frequency limit of Jovian radio emissions and implications on source locations and Io plasma wake. Planetary Space Science, 49, 1137-1149. doi: 10.1016/S0032-0633(01)00021-6 